\title{
Disproportionate entrance length in superfluid flows and the puzzle of counterflow instabilities
}

\author{
J. Bertolaccini \\ Université Lyon, ENS de Lyon, CNRS, Laboratoire de Physique, F-69342 Lyon, France
}

\author{
E. Lévêque \\ Université Lyon, Ecole Centrale de Lyon, CNRS, Laboratoire de Mécanique des Fluides et d'Acoustique, \\ F-69134 Ecully cedex, France
}

\author{
P.-E. Roche \\ Institut NEEL, CNRS, and Université Grenoble Alpes, F-38042 Grenoble, France
}

(Received 25 July 2017; published 26 December 2017)

\begin{abstract}
Systematic simulations of the two-fluid model of superfluid helium (He-II) encompassing the Hall-Vinen-Bekharevich-Khalatnikov (HVBK) mutual coupling have been performed in two-dimensional pipe counterflows between 1.3 and $1.96 \mathrm{~K}$. The numerical scheme relies on the lattice Boltzmann method. A Boussinesq-like hypothesis is introduced to omit temperature variations along the pipe. In return, the thermomechanical forcings of the normal and superfuid components are fueled by a pressure term related to their mass-density variations under an approximation of weak compressibility. This modeling framework reproduces the essential features of a thermally driven counterflow. A generalized definition of the entrance length is introduced to suitably compare entry effects (of different nature) at opposite ends of the pipe. This definition is related to the excess of pressure loss with respect to the developed Poiseuille-flow solution. At the heated end of the pipe, it is found that the entrance length for the normal fluid follows a classical law and increases linearly with the Reynolds number. At the cooled end, the entrance length for the superfluid is enhanced as compared to the normal fluid by up to one order of magnitude. At this end, the normal fluid flows into the cooling bath of He-II and produces large-scale superfluid vortical motions in the bath that partly re-enter the pipe along its sidewalls before being damped by mutual friction. In the superfluid entry region, the resulting frictional coupling in the superfluid boundary layer distorts the velocity profiles toward tail flattening for the normal fluid and tail raising for the superfluid. Eventually, a simple analytical model of entry effects allows us to re-examine the long-debated thresholds of $T 1$ and $T 2$ instabilities in superfluid counterflows. Inconsistencies in the $T 1$ thresholds reported since the $1960 \mathrm{~s}$ disappear if an aspect-ratio criterion based on our modeling is used to discard data sets with the strongest entry effects. Furthermore, it is observed that entry effects can spuriously reproduce the signature of a $T 2$ transition with a normal flow remaining laminar.
\end{abstract}

DOI: 10.1103/PhysRevFluids.2.123902

\section{INTRODUCTION}

Viscosity plays a key role in determining the velocity profile of laminar flows in pipes. As the fluid enters the pipe, its loses memory of its entry velocity profile within a so-called entrance length that is inversely proportional to its kinematic viscosity. What happens when an inviscid fluid such as superfluid helium enters a pipe? This paper explores the concept of entrance length in superfluids and proposes an explanation of a decades-old controversy in superfluid hydrodynamics, namely, the inconsistencies in critical velocities associated with $T 1$ and $T 2$ instabilities of thermally driven superfluid counterflows in pipes and channels. 


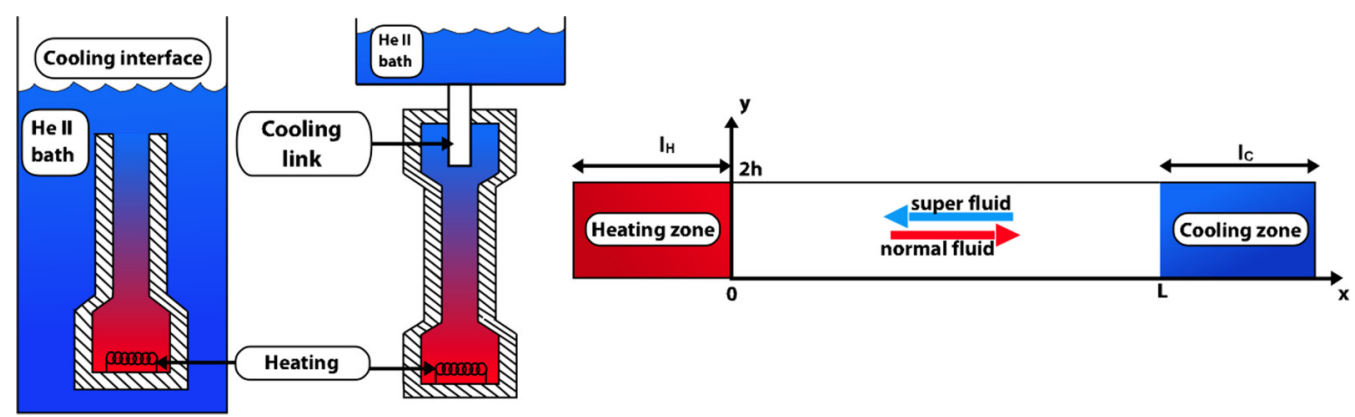

FIG. 1. Left-side and center sketches: Typical superfluid thermal counterflow experimental setups. For the sake of visibility, the pipe diameter has been exaggerated. Right-side sketch: Two-dimensional geometry of our numerical simulations.

\section{A. Critical velocities in superfluid counterflows}

The tricky instabilities of superfluid flows have stimulated research for more than 50 years. Probably the simplest and most studied flow involves a pipe filled with liquid ${ }^{4} \mathrm{He}$ in its superfluid state, usually referred to as He-II, with a closed end equipped with a heater and opened to a cooled bath of He-II at the other end. When a current is supplied to the heater, a flow-called "thermal counterflow" for reasons recalled later-naturally establishes in the pipe (see Ref. [1] for a seminal paper). In this flow, two dynamical instabilities called $T 1$ and $T 2$ have been observed [2,3].

According to Tisza and Landau's two-fluid model of He-II, a counterflow may be viewed as two mutually interpenetrating fluids flowing in opposite directions in the duct [4,5]. The so-called normal fluid is viscous and carries all the entropy of ${ }^{4} \mathrm{He}$ along one direction, whereas the superfluid moves in the opposite direction. The superfluid behaves like a fluid with zero viscosity and has no vorticity except along quantized vortex lines. The presence of these vortex lines is responsible for a mutual friction between the two fluids. We shall see later that this friction force is a key ingredient in the dynamics of counterflows.

When heat is supplied at the closed end of the pipe, the superfluid flows toward it to gain the heat content and transform itself into normal fluid. The normal fluid then flows away from the heater to evacuate heat (or entropy) toward the bath of He-II where the reverse conversion takes place, as sketched in Fig. 1. A stationary regime establishes itself, in which the averaged flux of normal fluid $V_{n}$ is determined by the heat flux (across a unit area) $W$ transferred throughout the pipe from the heater to the bath: $W=S T \times V_{n}$, where $S$ denotes the entropy per unit volume of ${ }^{4} \mathrm{He}$ and $T$ is the temperature of the thermostat. On the other hand, the averaged superfluid flux results from the conservation of mass, i.e., $\rho_{n} V_{n}+\rho_{s} V_{s}=0$ with obvious notations.

It has been widely accepted since the 1960 s [6] that the $T 1$ instability refers to the appearance of unsteadiness in the superfluid flow, whereas the normal-fluid flow remains laminar until the $T 2$ transition. A comprehensive review of the numerous models to describe the threshold of the $T 1$ instability is clearly beyond the scope of this paper; however, it is worth referring to a few of them to illustrate the level of controversy and, sometimes, confusion about this transition. For instance, the critical superfluid velocity $V_{c}$ of the $T 1$ instability is predicted to have either no dependence on the pipe diameter or channel hydraulic diameter $d$ [7-9] or a $d^{-1 / 4}$ dependence [10] or a $d^{-1 / 3}$ dependence with or without a logarithmic correction $[11,12]$ or a $d^{-1}$ dependence with a logarithmic correction [13-19] or, at last, a pure $d^{-1}$ dependence [20-22]. The models that predict no dependence on the diameter clearly overestimate the critical velocity but all the others reach reasonable degrees of agreement with some subsets of experimental data. For instance, the data compiled in Ref. [23] are found compatible with the three models predicting $d^{-1 / 4}$ and $d^{-1 / 3}$ dependencies, while the data reported by Ref. [24] better support a $1 / d$ dependence. Finally, the data in Ref. [4] reasonably agree with a $\log d / d$ dependence. 


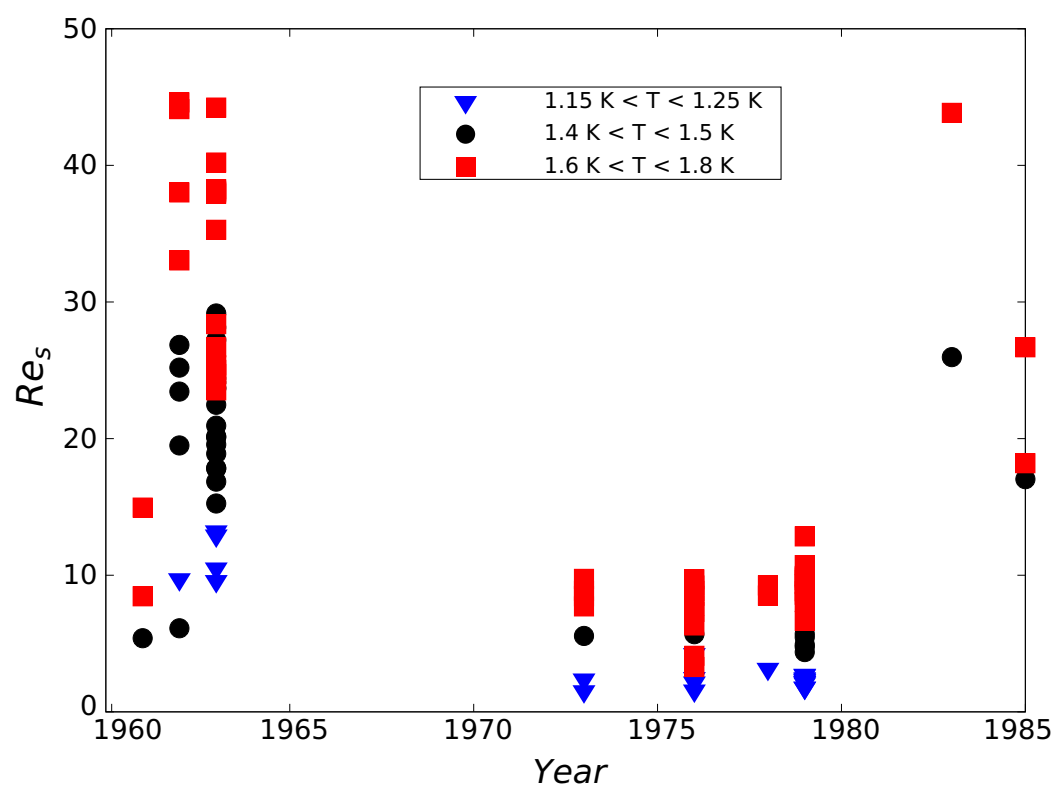

FIG. 2. Threshold of $T 1$ instability measured in various experiments of superfluid thermal counterflows. Superfluid critical Reynolds number $\operatorname{Re}_{s}=V_{c} d / \kappa$ vs year of publication of datasets (references are given in the text); $d$ is the hydraulic diameter of the channel or pipe.

To illustrate the scatter of experimental results, let us consider the models consistent with $V_{c} \sim d^{-1}$ suggesting that the transition is simply controlled by a critical superfluid Reynolds number $V_{c} d / \phi(T)$, where $\phi(T)$ is a modeled function of the temperature. In Fig. 2, the critical Reynolds $\operatorname{Re}_{s}=V_{c} d / \kappa$ with $\kappa$ being the quantum of circulation of ${ }^{4} \mathrm{He}$ is displayed for the datasets reported in ten papers published over a period of 25 years between 1960 and 1985 [25-34]. The datasets have been partitioned into three temperature ranges to better evidence a possible temperature dependence. In rare cases, it cannot be excluded that the transition had been wrongly attributed to $T 1$ instead of $T 2$, as already discussed in Refs. [23,35]. However, this possibility should not affect the overall picture emerging from Fig. 2. The scatter of critical Reynolds numbers exceeds one and a half decades. Obviously, the temperature dependence cannot explain such dispersion. The picture is not improved either if quantities such as the critical velocity or heat flux density are plotted on the vertical axis, or if the relative velocity between both fluids is used to define a Reynolds number. Therefore, it may be fairly claimed that the scatter of published experimental datasets does not allow us today to discriminate between models. It will be shown in this article that the pipe aspect ratio has been underestimated as an crucial design parameter in many experiments dedicated to the $T 1$ transition.

Finally, let us mention that such a confused situation is not restricted to the $T 1$ transition in pipes and channels but also applies to the $T 2$ transition, as discussed for instance in Refs. [2,6,22,25,35,36] and other superfluid transitions reported in pipes, channels, and flows through apertures with various types of thermomechanical forcing; e.g., see Refs. [37-41] and references therein. In Subsec. V B, the specific case of the $T 3$ transition is discussed. The $T 3$ transition is believed to replace the $T 1$ and $T 2$ transitions in channels with an elongated cross section.

\section{B. Entrance effects in pipe flows}

In the laminar regime, viscosity plays a central role in imposing itself on the velocity profile of a (classical) fluid entering a pipe, regardless of the profile at the entry. After a few tens of diameter, a characteristic parabolic profile establishes itself as the balance between the pressure gradient and 
the viscous shear stress. The no-slip condition is satisfied at the wall. Once this profile is developed, it no longer varies along the flow direction. The length of pipe required to reach this asymptotic profile or, equivalently, to lose the memory of the entry profile, is called the "entrance length" or "entry length."

To the best of our knowledge, there is no previous study of entrance effects in superfluid flows, even though the possible importance of this effect had been mentioned as early as 1957 by Vinen [1]. The possibility of entrance effects associated with boundary layer development has also been explicitly pointed out in Ref. [42]. Still, few indirect evidence that entrance effects could play a significant role in superfluid counterflows has been reported in the literature. For instance, an increase of the critical superfluid velocity by a factor of 1.3 has been observed after modifying the geometry of the end of the hottest tube [43]. Also, geometrical discontinuities at the superfluid inlet have been identified as a source of superfluid vorticity that is eventually carried far into the pipe $[44,45]$. Some authors noticed that their results could vary when changing the length of the pipe and, concomitantly, the shape of the entrance orifices. That way, a change of the critical velocity by almost a factor of 2 has been reported in Ref. [29], whereas "substantial discrepancy between results obtained with long and short tubes" has been mentioned in Ref. [38].

More generally, the inlet and outlet boundary conditions, e.g., the pipe opening into a bath, into a bulb, or abruptly closed, are expected to act as a source of vorticity associated with some recirculation of the flow. Such recirculation motions near the ends of the pipe have already been evidenced in numerical simulations $[46,47]$. In our study, we shall see that these vortical motions can have a strong impact on the development of the flow inside the pipe. At the cooled end of the pipe, the surrounding superfluid vorticity is eventually swept along into the pipe by the superfluid current, what results in some entrance effects associated with the presence of superfluid boundary layers. In that case, the development of the superfluid flow inside the pipe is no longer governed by the viscosity (since the superfluid is inviscid) but by the mutual friction between the normal and superfluid currents. The normal-fluid velocity profile is strongly affected in this region and an enhanced pressure drop is experienced.

\section{A preliminary qualitative result}

As will be shown later, the entrance length of a superfluid may be significantly larger than that of a classical fluid. To limit artifacts due to entry effects, the pipe or channel should therefore have an aspect ratio much larger than what would be expected from classical hydrodynamics. In Fig. 3, the superfluid critical Reynolds number $\operatorname{Re}_{s}$ related to the $T 1$ instability is now displayed as a function of the pipe (or channel) aspect ratio $L / d$ for the same datasets as in Fig. 2. Interestingly, it is found that all datasets obtained with aspect ratios larger than 550 are consistent between each other, while datasets with aspect ratios smaller than 140 exhibit scattered critical Reynolds numbers. This first important finding strongly supports the idea that superfluid entry effects could explain, at least partly, the scatter of thresholds of the $T 1$ instability reported during the past 50 years. It will be argued later that an aspect ratio of several hundreds is indeed required to safely escape from entry effects.

\section{PHYSICAL AND NUMERICAL MODELING}

Beyond entrance effects, our study deals more generally with the development of superfluid boundary layers, where the effect of mutual friction is essential. One must admit that our understanding of boundary layers in superfluid flows is very limited. For instance, the "frictional role" played by residual quantized vortices anchored on miscroscopic asperities of the sidewalls (e.g., see Refs. $[39,48,49]$ ) is not fully elucidated. A wall model is therefore a priori needed to carry out numerical simulations. Another difficulty comes from the lack of a mathematical framework fully accounting for He-II dynamics, which therefore imposes a certain level of arbitrariness on the chosen dynamical equations [50]. In this section, an original approach based on the lattice Boltzmann method is introduced to represent the problem at a mesoscopic level intermediate between 


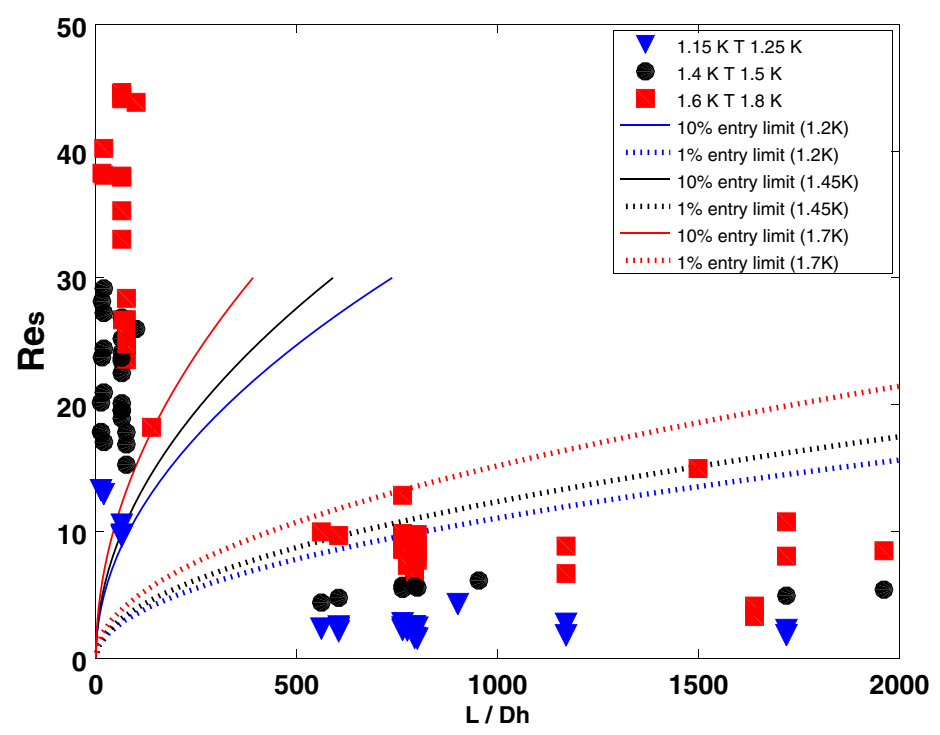

FIG. 3. The critical superfluid Reynolds number $\operatorname{Re}_{s}=V_{c} d / \kappa$ related to the $T 1$ transition is displayed as a function of the pipe aspect ratio $L / d$ for the same datasets as in Fig. 2. The continuous lines separate flow configurations with overall effective entry lengths contaminating more than $10 \%$ of the pipe length (upper left area) and less than $10 \%$ of the pipe length (lower right area). The dash lines mark similar separations with a $1 \%$ criterion. The lines arise from a simple analytical model detailed in Sec. III.

the microscopic and the macroscopic. In its continuous limit, this approach is compliant to the Hall-Vinen-Bekharevich-Khalatnikov (HVBK) model of He-II discussed in Sec. II B.

\section{A. The geometrical setting}

The left diagram in Fig. 1 shows the typical setup of a counterflow experiment as used, for instance, in Ref. [21]. In this experimental apparatus, the cooling is achieved by evaporation at the liquid-vapor interface, whereas the counterflow is activated by Joule heating in a thermally insulated bulb at the bottom end of the (insulated) pipe. For the sake of visibility, the diameter of the pipe has been exaggerated. It is actually much smaller than the dimensions of the He-II bath and of the heating bulb, so that the velocities reached inside the pipe are much larger than outside. A more symmetric arrangement is sometimes used by integrating a cooling bulb at the top end, as illustrated by the middle diagram in Fig. 1; e.g., see Ref. [34].

In our simulations, a simplified two-dimensional geometry for a closed pipe has been chosen (see Fig. 1). The heating and cooling processes are confined to two symmetric zones, which allows us to compare directly the entrance effects at opposite ends. The use of a spatially distributed heater and cooler mimics (to some degree) the real boundary conditions and favors the numerical stability of the simulations, as already argued in Ref. [51]. Finally, the rectangular geometry of the boundary is here idealized and does not exhibit any discontinuity or irregularity. These conditions are therefore much more favorable to the stability of the flow as compared to many experiments. In classical hydrodynamics, a two-dimensional approach captures the main phenomenology of entrance effects, at least in the steady regime before turbulence occurs. Accordingly, our simulations have been performed in two dimensions, which also reduces the computational cost and makes possible a systematic study. In Subsec. V B, we discuss the relevance of this two-dimensional (2D) model for the three-dimensional (3D) channels of various cross-sectional aspect ratios. 


\section{B. The governing equations under a Boussinesq-like hypothesis}

Our modeling relies on the Hall-Vinen-Bekharevich-Khalatnikov (HVBK) model, in which the assembly of quantized superfluid vortices is accounted for at a coarse-grained level by a continuous (nonzero) superfluid vorticity field [52]. This model is no longer justified when superfluid vortices are very scarce, or when vortices result from intrinsic nucleation rather than from the stretching of residual vortices attached to the sidewalls, or carried from the reservoirs. Such nucleation-limited flow regime can appear in highly controlled environments [41,53,54], which are not relevant in counterflow experiments. In particular, the HVBK model would not have been a priori justified if our interest was the initiation of the $T 1$ transition in a quiescent flow. Here, the conditions are different since our focus is on the persistence of pre-existing superfluid vortices entering the pipe from the reservoir.

The HVBK model is advantageous in that it allows us to account self-consistently for the mutual coupling between the superfluid and normal-fluid components. Therefore, it does not require any empirical assumption on the flow. Let us mention that such requirement is more difficult to fulfill [55] in vortex line (or vortex point) simulations of counterflows [18,56-62]. Finally, the HVBK model has already served as a mathematical basis for a number of previous numerical counterflow studies, e.g., see Refs. [5,36,46,51,63,64] and references therein.

The HVBK model may be viewed as a generalization of the two-fluid model introduced by Landau and Tisza [7,65]. It encompasses a mutual friction force $\mathbf{F}_{\mathrm{hvbk}}$ between the normal-fluid and the superfluid components. The model also accounts for an energy per unit length associated with the deformation of the vortex lines, which yields a vortex tension force acting on the superfluid component. The tension force is essential when considering rotating flows (with fully polarized quantized vortices) such as Taylor-Couette flows [66] but will be neglected in the present flow configurations. The resulting equations of motion for the normal fluid and superfluid therefore read

$$
\begin{aligned}
\rho_{n}\left[\frac{\partial \mathbf{v}_{n}}{\partial t}+\left(\mathbf{v}_{n} \cdot \nabla\right) \mathbf{v}_{n}\right] & =-\nabla p_{n}+\mu_{n} \nabla^{2} \mathbf{v}_{n}+\mathbf{F}_{\mathrm{hvbk}}, \\
\rho_{s}\left[\frac{\partial \mathbf{v}_{s}}{\partial t}+\left(\mathbf{v}_{s} \cdot \nabla\right) \mathbf{v}_{s}\right] & =-\nabla p_{s}-\mathbf{F}_{\mathrm{hvbk}}
\end{aligned}
$$

where $\mu_{n}$ is the dynamic viscosity of the normal fluid. The volumic forces $-\nabla p_{n}$ and $-\nabla p_{s}$ refer to generalized pressure (or chemical potential) gradients that encompass thermomechanical effects for the two components of He-II, respectively. Specifically, $\nabla p_{n}=\left(\rho_{n} / \rho\right) \nabla p+\left(\rho_{s} / \rho\right) S \nabla T$ and $\nabla p_{s}=\left(\rho_{s} / \rho\right) \nabla p-\left(\rho_{s} / \rho\right) S \nabla T$ with $\rho=\rho_{n}+\rho_{s}, p=p_{n}+p_{s}$, and $S$ being the total mass density, pressure, and entropy (per unit volume) of He-II. In principle, these dynamical equations must be supplemented by continuity equations for mass

$$
\begin{gathered}
\frac{\partial \rho_{n}}{\partial t}+\nabla \cdot\left(\rho_{n} \mathbf{v}_{n}\right)=\frac{\rho_{n} w}{T S}, \\
\frac{\partial \rho_{s}}{\partial t}+\nabla \cdot\left(\rho_{s} \mathbf{v}_{s}\right)=-\frac{\rho_{n} w}{T S},
\end{gathered}
$$

where $w$ denotes the algebraic heat power (per unit volume) supplied locally. In addition, continuity equations for thermal energy (or entropy) and equations of state for both fluids relating $\rho_{n}, \rho_{s}, p$, and $T$ should be considered. In the following, we shall seek for a simplified formulation of counterflow dynamics, which nevertheless preserves its essential physical features.

In most counterflows, temperature and pressure variations along the pipe remain negligible compared to the temperature and pressure imposed by the helium bath. Besides, dissipation within the pipe is significantly smaller than the heating and cooling power at both ends. As a result, the relative variations of mass densities $\rho_{n}$ and $\rho_{s}$ along the pipe are also very small. Most often, the velocities $v_{n}$ and $v_{s}$ remain substantially below the speeds of first and second sounds. Therefore, the kinetics may be decoupled from the thermodynamics in the spirit of the Boussinesq's approximation [67]. It may 
be assumed in addition that the algebraic heat supply entering in the mass-conservation equations refers uniquely to the heating and cooling occurring in the reservoirs; i.e., the "self-heating" of He-II due to viscous and mutual friction dissipation is obviously neglected. Moreover, it is considered that these heat supply or removal act as sources and sinks of normal fluid and superfluid that naturally set the two fluids into opposite motions. This approach allows us to remove consistently all explicit temperature dependence while preserving the counterflow thermal driving.

To solve this modeled dynamics using the lattice Boltzmann method, the incompressibility condition is approached in the weak-compressibility limit for both isothermal components of He-II. This expresses formally for the normal fluid as

$$
\delta p_{n}(\mathbf{x}, t)=c_{s}^{2} \delta \rho_{n}(\mathbf{x}, t),
$$

where $\delta \rho_{n}(\mathbf{x}, t)=\rho_{n}(\mathbf{x}, t)-\rho_{n}^{0}(T)$ with $\rho_{n}^{0}(T)$ being the mass density at rest at temperature $T$ and $\left|\delta \rho_{n}\right| / \rho_{n}^{0} \ll 1$, and similarly for the superfluid

$$
\delta p_{s}(\mathbf{x}, t)=c_{s}^{2} \delta \rho_{s}(\mathbf{x}, t)
$$

with $\delta \rho_{s}(\mathbf{x}, t)=\rho_{s}(\mathbf{x}, t)-\rho_{s}^{0}(T)$ and $\left|\delta \rho_{s}\right| / \rho_{s}^{0} \ll 1$. The velocity $c_{s}$ appears here as an arbitrary speed of sound that should be chosen much larger than the fluid velocities, i.e., $\left|v_{n}\right|$ and $\left|v_{s}\right| \ll c_{s}$. In this low Mach limit, the relative variations of $\rho_{n}$ and $\rho_{s}$ are expected to be very small $\left[\sim O\left(\mathrm{Ma}^{2}\right)\right]$, consistent with our assumption of weak compressibility. In each reservoir, the conversion of one fluid into the other will produce opposite density fluctuations. The resulting pressure fluctuations [given by Eqs. (3) and (4)] will propagate at high velocity $c_{s}$ across the channel and eventually result in a macroscopic driving of the two fluids in opposite directions.

We shall see in the following that this simplified modeling indeed allows us to reproduce the main dynamical features of thermally driven counterflows. Furthermore, the particulate nature of the lattice Boltzmann method will be particularly adequate to model the local conversion rate between the normal and superfluid particles.

\section{The mutual friction}

The modeling of the mutual friction in a counterflow is a delicate issue $[22,66,68-70]$. Here, the standard HVBK formulation has been adopted without the vortex tension term. Namely,

$$
\mathbf{F}_{\mathrm{hvbk}}=\frac{B}{2} \frac{\rho_{n} \rho_{s}}{\rho} \hat{\omega}_{\mathbf{s}} \times\left(\omega_{\mathbf{s}} \times \mathbf{v}_{\mathbf{n s}}\right)+\frac{B^{\prime}}{2} \frac{\rho_{n} \rho_{s}}{\rho}\left(\omega_{\mathbf{s}} \times \mathbf{v}_{\mathbf{n s}}\right),
$$

where $\omega_{\mathbf{s}} \equiv \nabla \times \mathbf{v}_{\mathbf{s}}$ is the coarse-grained superfluid vorticity field with $\hat{\omega}_{\mathbf{s}} \equiv \omega_{\mathbf{s}} /\left|\omega_{\mathbf{s}}\right|$. The friction velocity is defined by $\mathbf{v}_{\mathbf{n s}} \equiv \mathbf{v}_{\mathbf{n}}-\mathbf{v}_{\mathbf{s}} ; B$ and $B^{\prime}$ are temperature-dependent friction coefficients of order 1 . Let us mention that our interest in the primary instability of the flow, below the $T 1$ transition but in the presence of residual superfluid vortices originating from the reservoirs, a priori excludes the Gorter-Mellink force [5] that is expected to hold only above the $T 1$ transition with self-sustained vorticity in the pipe. Even though the HVBK force has been originally derived to deal with rotating cryostat experiment, its relevance for pipe flow has already been pointed out in Ref. [71].

Our main concern is to investigate entrance effects and, especially, the persistence of superfluid vorticity entering from the cooled end of the pipe. This superfluid vorticity is expected to influence the coupled dynamics of the counterflow through the HVBK force.

\section{The numerical method}

\section{The lattice Boltzmann scheme}

For the first time, the lattice Boltzmann (LB) method has been used to simulate a flow of superfluid helium. The LB method offers a computationally efficient particle-based alternative to conventional continuum-based approaches to simulate fluid dynamics [72]. The fluid is considered at a mesoscopic level. More precisely, the fluid is viewed as populations of particles that collide, redistribute, and 


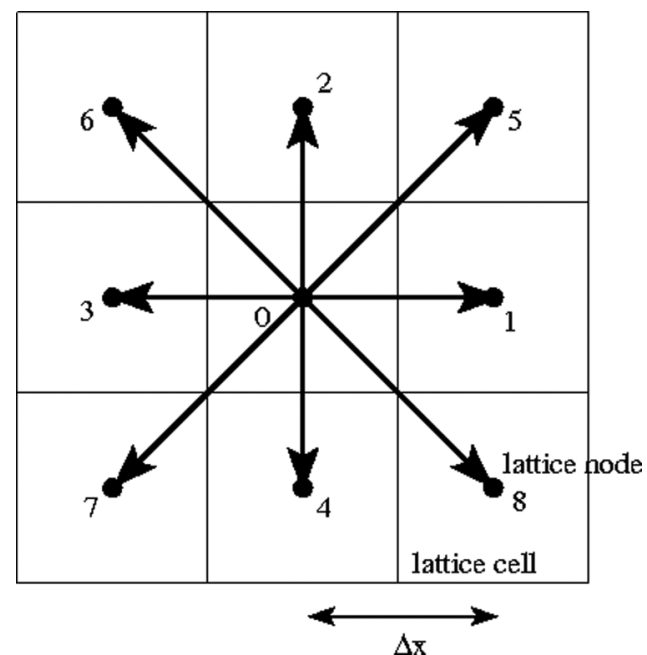

FIG. 4. Sketch of the D2Q9 lattice in a cell-centered representation. During one time step, particles move exactly from a lattice node toward one of its nine neighbours (including the node itself) and collide. By nature, the lattice spacing is related to the time step by $\Delta x / \Delta t=\sqrt{3} c_{s}$, where $c_{s}$ characterizes the speed of particles on the lattice.

propagate along the different links of a discrete lattice. The complexity of the flow emerges from the repeated application of simple rules of streaming and collision at each lattice node. The macroscopic flow variables are recovered locally by averaging over the populations of particles moving with the different velocities. This approach obviously refers to the kinetic theory of fluids and rigorous connections can be established with the Boltzmann equation [73].

In the framework of the two-fluid model, the dynamics of He-II can be naturally accounted for by two interacting sets of populations associated respectively with the normal and superfluid components. The evolution of these populations is driven by a system of two coupled schemes encompassing the mutual HVBK force. This latter is considered as an external force in the momentum budget of each component. In order to circumvent the inappropriateness of the LB approach for inviscid fluid, an arbitrary small kinematic viscosity has been assigned to the superfluid component. In practice, a ratio $v_{s} / v_{n}=0.01$ has been used in all simulations where $v_{s}=\mu_{s} / \rho_{s}$ and $v_{n}=\mu_{n} / \rho_{n}$. This ratio ensures that the resulting viscous force remains subdominant in the dynamics of the superfluid component (as detailed later).

For simplicity, the LB scheme is now detailed for one component of the fluid only. It applies in the same manner for the other component with the opposite coupling force. The so-called D2Q9 lattice with nine different possible velocities has been adopted in two dimensions (see Fig. 4). An iteration proceeds in two steps: streaming and collision. First, "streaming" carries the collided populations $\mathrm{f}_{\alpha}^{\text {coll }}$ to neighboring lattice nodes according to their own velocity $\mathbf{c}_{\alpha}$ :

$$
f_{\alpha}\left(\mathbf{x}+\mathbf{c}_{\alpha} \Delta t, t+\Delta t\right)=f_{\alpha}^{\text {coll }}(\mathbf{x}, t)
$$

with $\Delta t$ being the time step. Second, "collision" redistributes instantaneously the incoming particles along the different directions of propagation:

$$
f_{\alpha}^{\text {coll }}(\mathbf{x}, t)=f_{\alpha}(\mathbf{x}, t)-\frac{1}{\tau}\left[f_{\alpha}-\mathrm{f}_{\alpha}^{\mathrm{eq}}\left(\rho, \mathbf{v}, \mathbf{F}_{\text {hvbk }}\right)\right](\mathbf{x}, t) .
$$

In the collision, the so-called Bhatnagar-Gross-Krook (BGK) approximation [74] $-\left(f_{\alpha}-\mathrm{f}_{\alpha}^{\mathrm{eq}}\right) / \tau$ is used. This approximation simply expresses as a relaxation with parameter $\tau$ toward an equilibrium function evaluated at the macroscopic level. The relaxation parameter $\tau$ is related to the viscosity 
by $v=(\tau-1 / 2) c_{s}^{2} \Delta t$. The rationale behind this approximation is that most details hidden in the collision operator play no role when going on the continuous limit. It is therefore replaced with a much handier expression retaining only the basic features of fluid dynamics. In this respect, the collision term also encompasses here an extra momentum exchange related to the mutual force $\mathbf{F}_{\text {hvbk }}$ between the two components of He-II.

It is rather direct to establish through a Chapman-Enskog time-scale expansion [75] that the equilibrium function in Eq. (7) should develop as

$$
f_{\alpha}^{e q}\left(\rho, \mathbf{v}, \mathbf{F}_{\mathrm{hvbk}}\right)=w_{\alpha}\left[A+\frac{B_{i} c_{\alpha i}}{c_{s}^{2}}+\frac{C_{i j}\left(c_{\alpha i} c_{\alpha j}-c_{s}^{2} \delta_{i j}\right)}{2 c_{s}^{4}}\right]
$$

by using Einstein summation convention, with

$$
\begin{aligned}
A & =\rho, \\
B_{i} & =\rho v_{i}+v / c_{s}^{2} F_{\mathrm{hvbk}_{i},} \\
C_{i j} & =\rho v_{i} v_{j}+2 v / c_{s}^{2} F_{\mathrm{hvbk}_{i}} v_{j},
\end{aligned}
$$

to be compliant to the HVBK equations in the continuous limit. The derivation of this equilibrium function may be inspired from Ref. [76]. The weight factors are $w_{0}=4 / 9, w_{1 \ldots 4}=1 / 9$, and $w_{5 \ldots .}=$ 1/36 for the D2Q9 lattice. This LB scheme is isothermal and relies on the weak-compressibility assumption $\delta p=c_{s}^{2} \delta \rho$, which directly echoes the previous physical modeling. The characteristic velocity $c_{s}$ is identified as the speed of sound introduced before.

The macroscopic flow variables are obtained by integrating over the populations according to

$$
\begin{gathered}
\rho=\sum_{\alpha} f_{\alpha}, \\
\rho \mathbf{v}=\sum_{\alpha} f_{\alpha} \mathbf{c}_{\alpha}+\frac{\Delta t}{2} \mathbf{F}_{\text {hvbk }}
\end{gathered}
$$

This discrete scheme approximates the HVBK equations in the continuous limit with a third-order error in Mach number $\mathrm{Ma}=|\mathbf{v}| / c_{s}$. As already mentioned, $c_{s}$ has been fixed arbitrarily in our simulations to keep the Mach number sufficiently small and alleviate this error [77]. In practice, the maximum Mach number reached in our simulations is $\mathrm{Ma}_{\max }=0.025$ at the highest Reynolds numbers. Finally, let us mention that the standard BGK collision term has been eventually replaced by an equivalent central-moment collision operator, as detailed in Refs. [78,79], in order to enhance the numerical stability of the scheme.

The density can be evaluated directly from the distributions according to Eq. (8). In contrast, the additional term $\Delta t / 2 \mathbf{F}_{\text {hvbk }}$ in Eq. (9) does not allow us to compute explicitly the velocity since $\mathbf{F}_{\text {hvbk }}$ depends itself on the friction velocity $\mathbf{v}_{\mathbf{n s}}=\mathbf{v}_{\mathbf{n}}-\mathbf{v}_{\mathbf{s}}$ and the superfluid vorticity $\omega_{s}=\nabla \times \mathbf{v}_{\mathbf{s}}$.

Instead, a nonlinear system must be solved to get $\mathbf{v}_{n}, \mathbf{v}_{s}$, and $\omega_{s}$ :

$$
\begin{aligned}
& \rho_{n} \mathbf{v}_{n}=\sum_{\alpha} f_{\alpha}^{(n)} \mathbf{c}_{\alpha}+\frac{\Delta t}{2} \mathbf{F}_{\mathrm{hvbk}}\left(\mathbf{v}_{n s}, \omega_{s}\right), \\
& \rho_{s} \mathbf{v}_{s}=\sum_{\alpha} f_{\alpha}^{(s)} \mathbf{c}_{\alpha}-\frac{\Delta t}{2} \mathbf{F}_{\mathrm{hvbk}}\left(\mathbf{v}_{n s}, \omega_{s}\right) .
\end{aligned}
$$

An iterative predictor-corrector scheme has been designed for this purpose. The superfluid vorticity is firstly predicted as $\omega_{s}^{\star}$ by a linear extrapolation of its values at the two previous time steps. Therefore, the HVBK force is predicted as $\mathbf{F}_{\mathrm{hvbk}}\left(\mathbf{v}_{n s}, \omega_{s}^{\star}\right)$. By including this expression in Eqs. (10) and (11), one obtains a linear system in $\mathbf{v}_{n}$ and $\mathbf{v}_{s}$ that can be easily inverted. Then, the superfluid vorticity is consistently corrected from second-order finite differences of the newly computed superfluid velocity: $\omega_{s}=\nabla \times \mathbf{v}_{s}$. This correction step is eventually repeated until a 

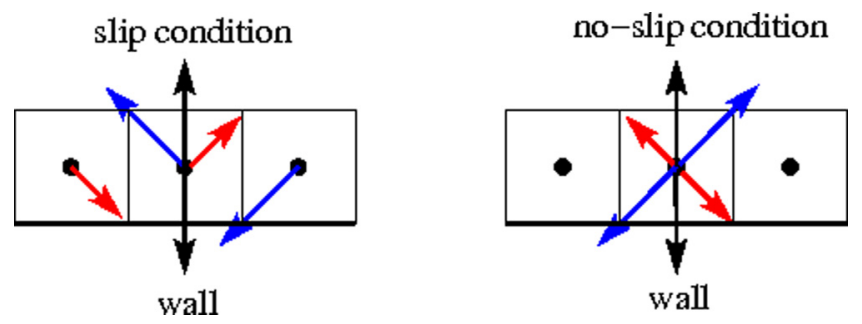

FIG. 5. The free-slip condition (superfluid) is accounted by a specular reflection of the particles hitting the wall, whereas a bounce back is considered for the no-slip condition (normal fluid). The wall is located half way between two lattice nodes.

convergence is obtained for the superfluid vorticity with a tolerance smaller than $10^{-4}$. As soon as the velocities and the superfluid vorticity are known, the equilibrium distribution $f_{\alpha}^{\mathrm{eq}}\left(\rho, \mathbf{v}, \mathbf{F}_{\mathrm{hvbk}}\right)$ can be evaluated for each component to fulfill the collision step. The application of the streaming step is straightforward.

\section{The boundary conditions}

In the flow geometry described in Fig. 1, solid boundaries are straight walls. The no-slip condition has been taken into account for the normal-fluid component by a standard half-way bounce-back procedure, which strictly ensures the conservation of mass and preserves the second-order accuracy of the LB scheme [72]. Consistently, a specular reflection of the particles hitting the wall has been considered for the superfluid component. For the specular reflection, the momentum transferred to the wall has only a component normal to the wall thus achieving a free slip (no tangential force). The specular reflection and the bounce back are sketched in Fig. 5.

\section{The forcing of the counterflow}

A crucial aspect of our approach is related to the driving of the counterflow at opposite ends of the channel. In this respect, the particulate nature of the LB approach appears to be adequate to treat the exchange of particles between the normal and superfluid populations in these zones.

It is physically expected that a local supply of heat induces a transfer of particles from the superfluid to the normal component. It is therefore natural to introduce an exchange coefficient $0 \leqslant$ $\gamma_{\text {heat }}<1$ to quantify the proportion of the superfluid particles being transferred to the normal-fluid component during one time step in one lattice cell. By convention, there is no heating for $\gamma_{\text {heat }}=0$. In the framework of the LB approach, the "heated populations" $\widetilde{f}_{\alpha}^{(n)}$ and $\widetilde{f}_{\alpha}^{(s)}$ are therefore updated according to

$$
\begin{aligned}
& \widetilde{f}_{\alpha}^{(s)}=\left(1-\gamma_{\text {heat }}\right) f_{\alpha}^{(s)}, \\
& \widetilde{f}_{\alpha}^{(n)}=f_{\alpha}^{(n)}+\gamma_{\text {heat }} f_{\alpha}^{(s)},
\end{aligned}
$$

at every time step and lattice node in the heating zone. To improve the stability and avoid strong compressibility disturbances, the heating has been considered only for the particles moving in the streamwise direction of the superfluid flow, i.e., $\alpha=3$. Similarly, the transfer of mass operates from the normal to the superfluid component with an exchange coefficient $\gamma_{\text {cool }}$ in the cooling zone. The "cooled populations" $\widetilde{f}_{\alpha}^{(n)}$ and $\widetilde{f}_{\alpha}^{(s)}$ are thus updated according to

$$
\begin{aligned}
& \widetilde{f}_{\alpha}^{(n)}=\left(1-\gamma_{\text {cool }}\right) f_{\alpha}^{(n)}, \\
& \widetilde{f}_{\alpha}^{(s)}=f_{\alpha}^{(s)}+\gamma_{\text {cool }} f_{\alpha}^{(n)},
\end{aligned}
$$


TABLE I. Dimension of the geometry sketched in Fig. 1 (in lattice units). At the highest Reynolds number, $L$ was increased up to 3000 to prevent merging of the entrance lengths (see Fig. 11).

\begin{tabular}{lcccc}
\hline \hline$L$ & $2 h$ & $L / 2 h$ & $l_{\mathrm{H}}$ & $l_{\mathrm{C}}$ \\
\hline 1800 & 60 & 30 & 200 & 200 \\
3000 & 60 & 50 & 200 & 200 \\
\hline \hline
\end{tabular}

with $\alpha=1$ corresponding to the streamwise direction of the normal-fluid flow. Here, $\gamma_{\text {cool }}$ is not free but is determined by the requirement to conserve the respective masses of normal fluid and superfluid inside the pipe. This constraint is imposed by the assumption of constant temperature of the system. Therefore, the exchange coefficient $\gamma_{\text {cool }}(t)$ is determined dynamically as

$$
\gamma_{\text {cool }}(t)=\gamma_{\text {heat }} \frac{\sum_{\mathbf{x} \in \mathcal{H}} f_{3}^{(s)}(\mathbf{x}, t)}{\sum_{\mathbf{x} \in \mathcal{C}} f_{1}^{(n)}(\mathbf{x}, t)},
$$

which ensures that the mass of superfluid lost in the heating zone $(\mathcal{H})$ is exactly recovered in the cooling zone $(\mathcal{C})$, and vice versa for the normal fluid.

It is important to mention that the heating and cooling processes are here idealized and designed to mimic the dynamical features of a thermal counterflow; it is not meant to reproduce the real physical processes. The modeling ensures the conservation of the overall mass of each component and a balance of mass fluxes consistent with the requirement that there is no net mass flux (in mean) in a pipe counterflow:

$$
\rho_{n} \mathbf{V}_{n}+\rho_{s} \mathbf{V}_{s}=\mathbf{0}
$$

where $\mathbf{V}_{n}$ and $\mathbf{V}_{s}$ denote the normal-fluid and superfluid velocities averaged over a cross section and time (or more precisely the averaged mass flux of each fluid normalized by its average density).

\section{Numerical settings}

From now on, numerical results are given in lattice units, i.e., based on the reference length scale $\Delta x$ (spacing of the lattice) and time scale $\Delta t$, with $\Delta x / \Delta t=\sqrt{3} c_{s}$ being imposed by the lattice dynamics (see Fig. 4). The geometry of the flow is sketched in Fig. 1. In our simulations, the aspect ratio of the duct is $L / 2 h=30$ and $L / 2 h=50$. The width $2 h=60$ has been kept constant. The extension of the heating and cooling zones is $l_{\mathrm{H}}=l_{\mathrm{C}}=200$. This setting is summarized for convenience in Table I. Counterflows are investigated at various temperatures ranging from 1.3 to $1.96 \mathrm{~K}$ (see Table II). For each temperature, the related physical constants have been set according to the reference values reported in Ref. [80]. In practice, the relaxation parameters have been kept constant with $\tau_{n}=0.5025$ and $\tau_{s}=0.500025$ for the normal fluid and the superfluid respectively, ensuring the stability of the scheme.

In counterflow experiments, the supplied heat flux $W$ (per unit area) is considered as the relevant control parameter of the flow. It determines the flow rate of the normal fluid inside the pipe according to $V_{n}=W / \mathrm{S} T$, where $S$ is the entropy per unit volume and, therefore, the Reynolds number based

TABLE II. Physical parameters of He-II at equilibrium for the investigated temperatures.

\begin{tabular}{lcccccc}
\hline \hline$T(\mathrm{~K})$ & $B$ & $B^{\prime}$ & $\rho\left(\mathrm{kg} / \mathrm{m}^{3}\right)$ & $\rho_{n} / \rho$ & $\rho_{s} / \rho_{n}$ & $\mu_{n}\left(10^{-6} \mathrm{Pas}\right)$ \\
\hline 1.3 & 1.526 & 0.616 & 145.12 & 0.045 & 21.22 & 1.528 \\
1.5 & 1.296 & 0.317 & 145.16 & 0.111 & 8.009 & 1.346 \\
1.7 & 1.100 & 0.107 & 145.27 & 0.229 & 3.37 & 1.290 \\
1.96 & 0.981 & 0.045 & 145.55 & 0.495 & 1.02 & 1.409 \\
\hline \hline
\end{tabular}




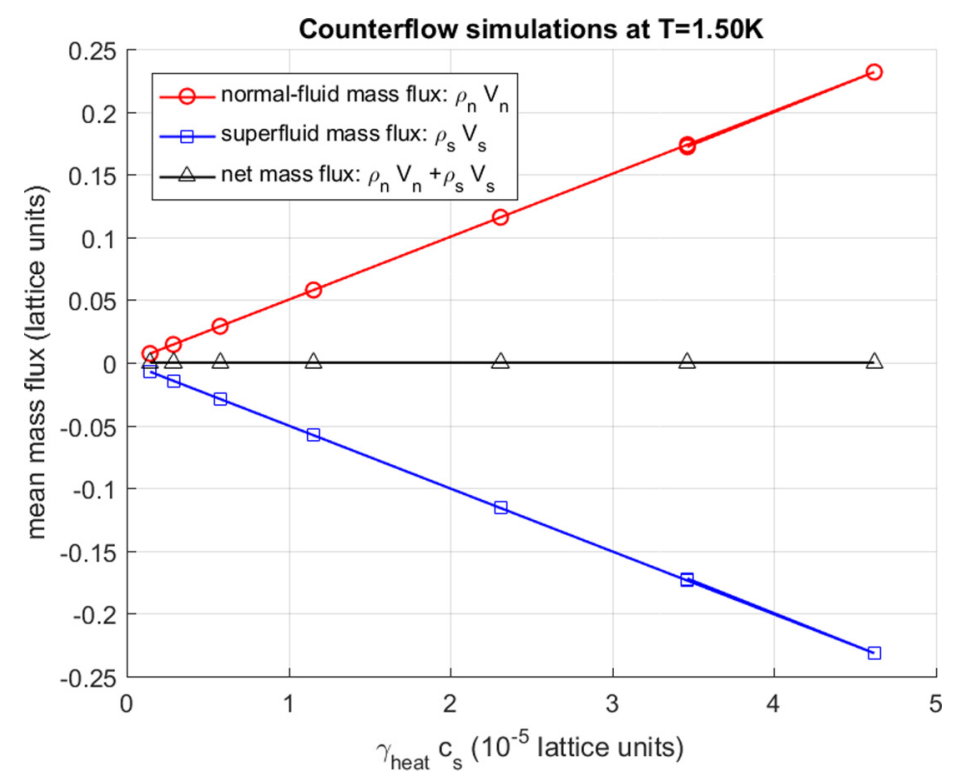

FIG. 6. Mean mass fluxes across a section in the center of the pipe for various values of the exchange coefficient at $T=1.5 \mathrm{~K}$. The net mass flux is null for all values of the exchange coefficient, which is the signature of a counterflow.

on the normal-fluid flow

$$
\operatorname{Re}_{\mathrm{n}}=\frac{\rho_{n} V_{n} 2 h}{\mu_{n}}
$$

The (averaged) mass fluxes at $T=1.5 \mathrm{~K}$ are displayed for various values of the exchange coefficient in Fig. 6. An obvious linear dependence is found for the normal-fluid component

$$
V_{n} \propto \gamma_{\text {heat }}
$$

This law can be seen as the analogous to $V_{n} \propto W$ at a given temperature. Moreover, $V_{s}$ also exhibits a linear dependence on the exchange coefficient so that the net mass flux $\rho_{n} V_{n}+\rho_{s} V_{s}$ vanishes. Figure 7 generalizes to different temperatures the heat flux analogy, showing that the opposite mass fluxes and the Reynolds numbers are controlled by the lattice parameter $\gamma_{\text {heat }}$ in a given geometry.

Formally, the mass conservation of normal fluid in the heating reservoir $(\mathcal{H})$ allows us to write in physical units that

$$
\left(\gamma_{\text {heat }} \sum_{\mathbf{x} \in \mathcal{H}} f_{3}^{(s)}(\mathbf{x})\right) \Delta x^{2} / \Delta t=\rho_{n} V_{n} 2 h .
$$

where $\rho_{n} V_{n}$ is the mean normal-fluid mass flux in the channel. Since heat (entropy) is carried by the normal fluid, $W=S T V_{n}$ and

$$
\gamma_{\text {heat }}=\frac{\rho_{n} W}{S T} 2 h \frac{1}{\sum_{\mathbf{x} \in \mathcal{H}} f_{3}^{(s)}(\mathbf{x})} \Delta t / \Delta x^{2} .
$$

This formal equation is exact and stems simply from mass conservation. In order to further highlight the correspondence between the exchange coefficient $\gamma_{\text {heat }}$ and physical quantities, an assumption on $\sum_{\mathbf{x} \in \mathcal{H}} f_{3}^{(s)}(\mathbf{x})$ is required. Therefore, we assume that the superfluid is nearly at statistical equilibrium in the heating reservoir and that compressibility corrections are negligible. 


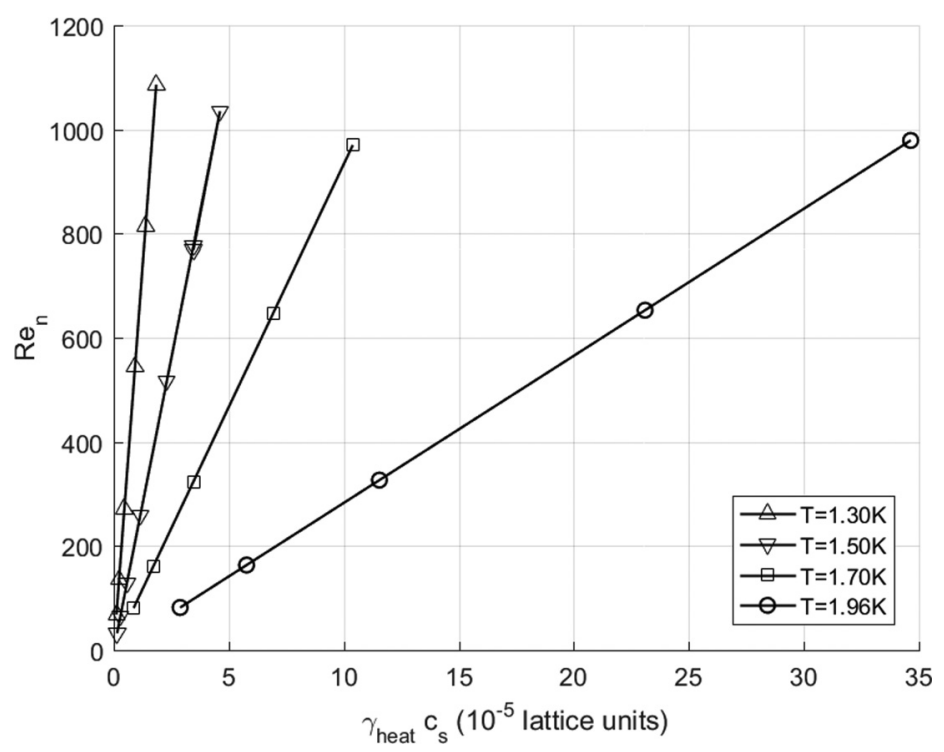

FIG. 7. The Reynolds number based on the normal-fluid flow, $\operatorname{Re}_{\mathrm{n}}=\rho_{n} V_{n} 2 h / \mu_{n}$, as a function of the exchange coefficient at investigated temperatures.

This leads to $\sum_{\mathbf{x} \in \mathcal{H}} f_{3}^{(s)}(\mathbf{x}) \simeq\left(\rho_{s} / 9\right) l_{\mathrm{H}} 2 h / \Delta x^{2}$ and, eventually, to

$$
\gamma_{\text {heat }} c_{s} \simeq \frac{\rho_{n}}{\rho_{s}} \frac{W}{S T} \frac{3 \sqrt{3}}{l_{\mathrm{H}} / \Delta x} \quad \text { with } \quad \frac{W}{S T} \equiv V_{n}
$$

by considering $\Delta x / \Delta t=\sqrt{3} c_{s}$. Equation (22) establishes a formal link between the exchange parameter $\gamma_{\text {heat }}$ entering in the lattice Boltzmann dynamics and the heating flux $W$ in real-world thermal counterflows. Its validity is now examined.

The zero-net-mass-flux condition $\rho_{n} V_{\mathrm{n}}+\rho_{s} V_{\mathrm{s}}=0$ allows us to rewrite Eq. (22) as

$$
\gamma_{\text {heat }} c_{s} \simeq\left|V_{s}\right| \frac{3 \sqrt{3}}{l_{\mathrm{H}} / \Delta x} \text {. }
$$

Figure 8 shows that this equation is well satisfied for all temperatures. Furthermore, by identifying $\left|V_{s}\right| / c_{s}$ with the superfluid Mach number, $\mathrm{Ma}_{\mathrm{s}}$, one obtains that the present modeling is expected to hold provided that

$$
\gamma_{\text {heat }} \ll \frac{3 \sqrt{3}}{l_{\mathrm{H}} / \Delta x}
$$

in order to fulfill the weakly compressibility condition $\left(\mathrm{Ma}_{\mathrm{s}} \ll 1\right)$. In our simulations $l_{\mathrm{H}}=200 \Delta x$, which yields $3 \sqrt{3} /\left(l_{\mathrm{H}} / \Delta x\right) \approx 0.026$. According to Fig. 8 , the exchange coefficient $\gamma_{\text {heat }}<610^{-4}$ since $c_{s}=1 / \sqrt{3}$ in lattice units. The condition Eq. (24) is therefore reasonably well satisfied in our simulations.

\section{A simplified test case}

At first, our code has been validated on a simplified case allowing us to check the accuracy of the numerical solution with respect to an analytical prediction. The impact of the artificial superfluid viscosity on velocity profiles has also been examined. 


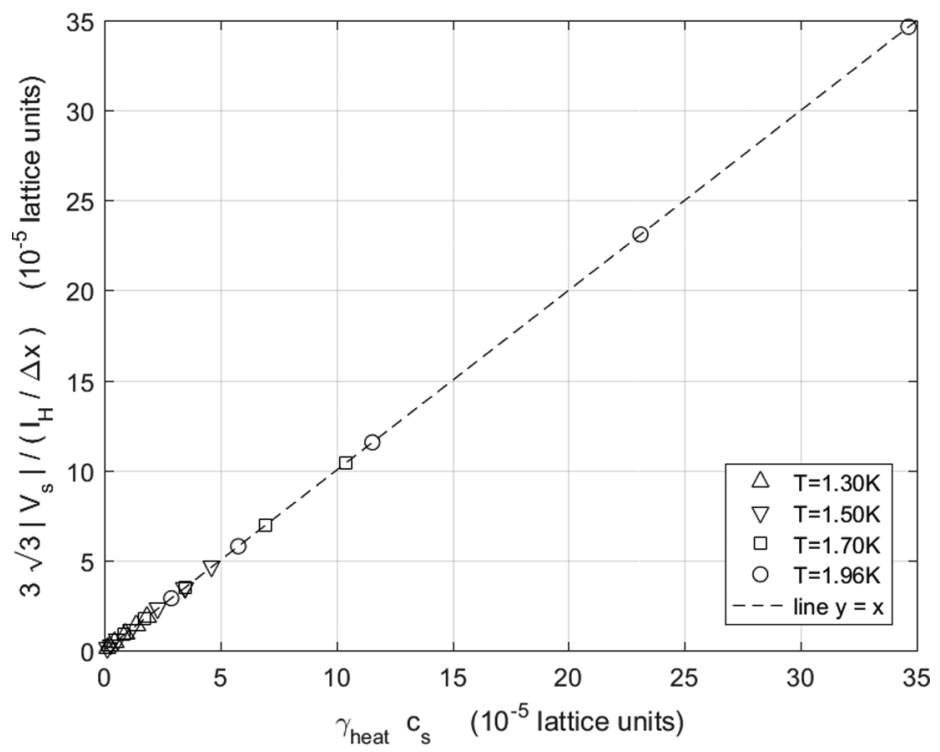

FIG. 8. The superfluid velocity within the duct is related to the lattice exchange coefficient according to $\left|V_{s}\right| 3 \sqrt{3} /\left(l_{\mathrm{H}} / \Delta x\right) \simeq \gamma_{\text {heat }} c_{s}$.

Specifically for this test case, the mutual friction force is simplified as

$$
\mathbf{F}_{\mathrm{hvbk}}=-\frac{B}{2} \frac{\rho_{n} \rho_{s}}{\rho} \Omega_{s} \mathbf{v}_{\mathrm{ns}}
$$

where $\Omega_{s}=10^{-4}$ (normalized by the inverse of the time step $\Delta t$ ) is here considered constant. Physical constants are taken at $T=1.50 \mathrm{~K}$. The Reynolds number of the simulated flow is $\operatorname{Re}_{\mathrm{n}}=65$. The flow is laminar for both components. The two developed velocity profiles (in the center of the duct) exhibit the same parabolic form with an offset. This is now detailed.

From the dynamical equations and by accounting for the artificial superfluid viscosity, the pressure gradient is constant in the duct with

$$
\nabla p=\mu_{\mathrm{eff}} \nabla^{2} \mathbf{v}_{\mathrm{n}}
$$

where the effective dynamical viscosity is given by

$$
\mu_{\mathrm{eff}}=\mu_{n}\left(1+\frac{\rho_{s}}{\rho_{n}} \frac{v_{s}}{v_{n}}\right) .
$$

The normal fluid therefore follows a parabolic velocity profile given by

$$
v_{n}(y)=\frac{h^{2} \nabla p}{2 \mu_{\mathrm{eff}}}\left[1-\left(\frac{y-h}{h}\right)^{2}\right],
$$

whereas the superfluid-velocity profile satisfies

$$
v_{s}(y)=v_{n}(y)-\frac{1}{\Omega_{s}} \frac{\rho}{\rho_{n} \rho_{s} B / 2}\left(\nabla p_{s}-\frac{\mu_{s}}{\mu_{\text {eff }}} \nabla p\right) .
$$

The numerical results obtained for $v_{s} / v_{n}=0.01$ are compared directly with the analytical predictions Eqs. (28) and (29) in Fig. 9. Let us note that the pressure gradients in these equations have been evaluated from the numerical solution. The overall agreement is excellent. Nevertheless, one may point out a small discrepancy at the first off-wall points for the superfluid component. 


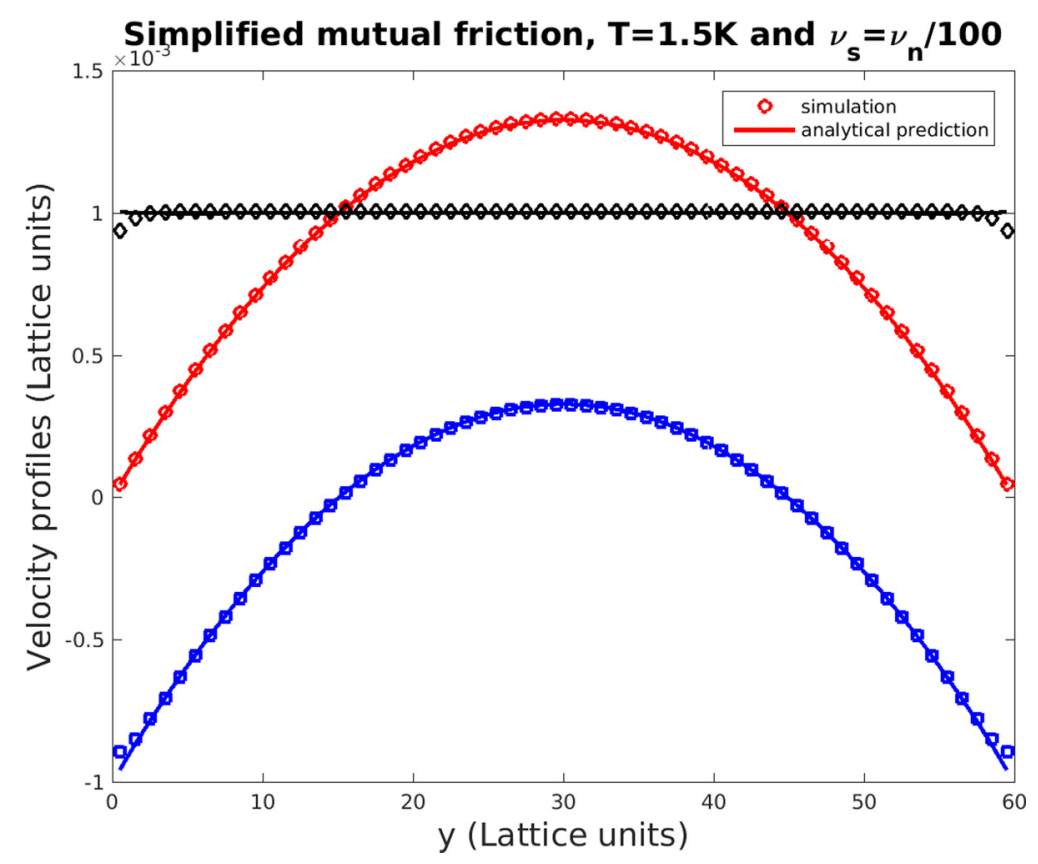

FIG. 9. Normal-fluid (red circles), superfluid (blue squares), and relative (blacks diamonds) velocity profiles in the center of the duct obtained from lattice Boltzmann simulation with the simplified mutual friction Eq. (25) at $1.5 \mathrm{~K}$. Comparisons are carried out with the analytical solutions (solid lines) given by Eqs. (28) and (29).

This slight deviation results from the physical mismatch between the slip condition, which prevents any transfer of tangential momentum to the wall and the presence of a small but nonzero viscous shear stress $v_{s}\left(d v_{s} / d y\right)$ at the wall. This effect is obviously an artifact arising from the finiteness of the superfluid viscosity. The influence of the artificial superfluid viscosity is further examined by considering the same test case with $v_{s} / v_{n}=0.002$ (see Fig. 10). The effect of the superfluid viscosity on the first off-wall points is reduced as expected from the above-mentioned analysis, but no notable difference is found for all other points. This indicates that the superfluid viscosity with $v_{s} / v_{n}=0.01$ has already very little influence on velocity profiles. In the next counterflow simulations, we will assume confidently that the superfluid viscosity has no notable effect on the observed velocity profiles.

\section{NORMAL-FLUID AND SUPERFLUID ENTRANCE LENGTHS}

In classical hydrodynamics, the entrance length is usually defined as the distance $L_{v}$ (or $L_{\tau}$ ) along which the centerline velocity (respectively, the shear stress at the wall) reaches $99 \%$ (resp., $98 \%$ ) of its asymptotic value in the fully developed regime, assuming a flat entry profile. The wall shear stress is highest at the pipe entrance and relaxes to a constant value in the fully developed region. The viscous shear stress at the wall then exactly balances the pressure gradient.

In the case of laminar flows, the entrance length normalized by the hydraulic diameter of the duct is found proportional to the Reynolds number of the flow $\operatorname{Re}_{D}=V D / v$ with

$$
\frac{L_{v}}{D}\left(\operatorname{resp} \cdot \frac{L_{\tau}}{D}\right) \simeq 0.05 \operatorname{Re}_{D}
$$

The previous definitions of entrance length are not suitable for counterflows due to the existence of two intricate fluids with different phenomenologies (not mentioning the experimental measurement difficulties in probing the centerline velocity or the shear stress at the wall). We shall rather propose 


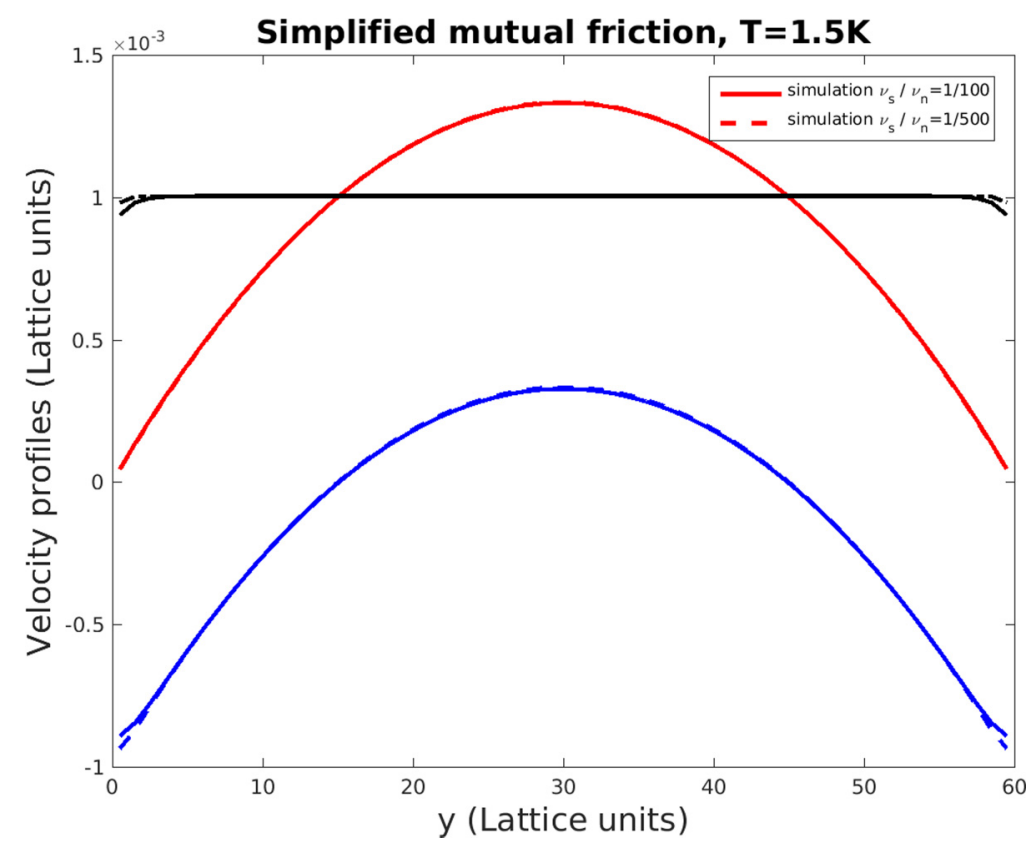

FIG. 10. Comparisons between numerical solutions obtained for $v_{s} / v_{n}=0.01$ and $v_{s} / v_{n}=0.002$ at $1.5 \mathrm{~K}$, with a simplified mutual friction. There is no notable difference between the profiles except for the first off-wall point for the superfluid.

a definition of the entrance length related to the excess of pressure drop caused by the resistive forces (viscosity and mutual friction) at each end of the pipe. Still, for the sake of a comparison with the classical case, the standard definitions will first be used with the normal component of He-II.

\section{A. Normal-fluid entrance length at the hot end}

The entrance length $L_{v}$ based on the normal-fluid velocity has been estimated at the heating end of the pipe for our simulations at $T=1.5 \mathrm{~K}$. The Reynolds number of the flow $\left(\mathrm{Re}_{\mathrm{n}}\right)$ varies from 32 to 1035 . The flow is laminar at the entrance and in the fully developed region. The dependence of $L_{v}$ on $\mathrm{Re}_{\mathrm{n}}$ is displayed in Fig. 11. The empirical law (30) valid for a flat velocity profile at the entry is also shown for comparison.

For low and moderate Reynolds numbers, $L_{v}$ grows linearly with $\operatorname{Re}_{\mathrm{n}}$ and agrees reasonably well with the empirical law. In our flow configuration, the normal-fluid velocity profile is not flat at the entry, as made evident in Fig. 12, which can explain why our estimations of $L_{v}$ are about $25 \%$ lower than standard values. A discrepancy from the linear growth also appears when $L_{v}$ reaches about $20 D$. In that case, our explanation is that the pipe is not long enough and that entrance effects originating from both ends eventually contaminate the whole pipe: There is no fully developed region, strictly speaking, but only a region where the two entrance regions merge. To corroborate this statement, a simulation in a longer tube with $L=3000$ and aspect ratio 50 (instead of 30) has been performed. The Reynolds number $\operatorname{Re}_{\mathrm{n}}=770$ and the value of $L_{v}$ is indicated by a filled symbol in Fig. 11 . We observe that the linear law is clearly recovered in that case.

In the fully developed region, the normal fluid follows a parabolic profile whereas the superfluid velocity is constant. In that case, it can be established from the dynamical equations that the pressure gradient must relate to the normal-fluid velocity (averaged over a section) according to

$$
p_{\infty}^{\prime} \equiv\left|\frac{d p_{\infty}(x)}{d x}\right|=\frac{3 \mu_{n} V_{n}}{h^{2}} .
$$




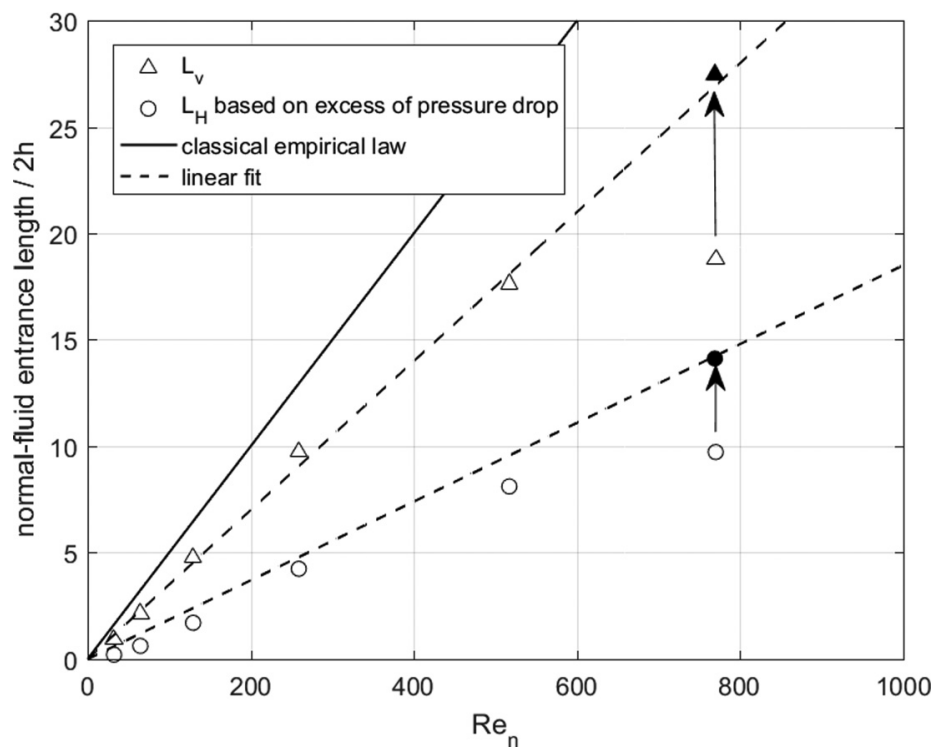

FIG. 11. Dimensionless entrance length of the normal fluid (at the hot end) at $T=1.5 \mathrm{~K}$. Two different estimations are used: $L_{v}$ relies on the centerline velocity, whereas $L_{\mathrm{H}}$ is based on the excess of pressure drop. Filled symbols indicate results obtained with a longer pipe of aspect ratio 50 (instead of 30) to avoid the merging of both entry effects.

This equation may be considered as a test of consistency for the achievement of a fully developed regime in the central region of the pipe. Its completion (within a relative error smaller than 10\%) has been checked systematically for all simulations at various temperatures and Reynolds numbers. For the simulations at $T=1.5 \mathrm{~K}$ in a pipe of aspect ratio 30 , Table III indicates that the fully developed regime was indeed not reached at the highest Reynolds numbers. These simulations have been ignored.

The agreement with the classical case may be understood simply. Below the $T 1$ transition, the superfluid that exits the pipe at the hot end does not carry a significant amount of vorticity. The superfluid velocity profile is flat in the entrance region of the normal fluid, as shown in Fig. 12. Therefore, the mutual coupling with the normal fluid is absent. Thus, the dynamics of the normal fluid follows Navier-Stokes equations and entrance effects are of classical nature.

We now introduce our alternative definition of the entrance length, inspired from the experimental method used to detect the transitions $T 1$ and $T 2$ from differential measurements across the reservoirs. This definition is based on the excess of pressure loss resulting from the action of the viscous and mutual friction forces in entry regions. The evolution of the averaged pressure $p(x)$ along the pipe is sketched in Fig. 13. In the fully developed region (central region of the pipe) the normal fluid follows a Poiseuille profile characterized by a linear pressure drop with $x$ referred to as $p_{\infty}(x)$ in Fig. 13. As a result of entrance effects, the pressure does not match the Poiseuille pressure $p_{\infty}(x)$ at $x=0$ and $x=L$ but there is an excess of pressure drop at each end. The entrance lengths $L_{\mathrm{H}}$ and $L_{\mathrm{C}}$ correspond to the equivalent length of pipe in the fully developed region, which would achieve the same excess of pressure drop. This is put in picture quite simply in Fig. 13.

By construction, $p_{\infty}\left(-L_{\mathrm{H}}\right)=p(0)$ and $p_{\infty}\left(L+L_{\mathrm{C}}\right)=p(L)$, which can be expressed equivalently as

$$
L_{\mathrm{H}}=\frac{p(0)-p_{\infty}(0)}{p_{\infty}^{\prime}}=\frac{1}{p_{\infty}^{\prime}} \int_{0}^{x_{\text {mid }}}\left[p^{\prime}(x)-p_{\infty}^{\prime}\right] d x
$$




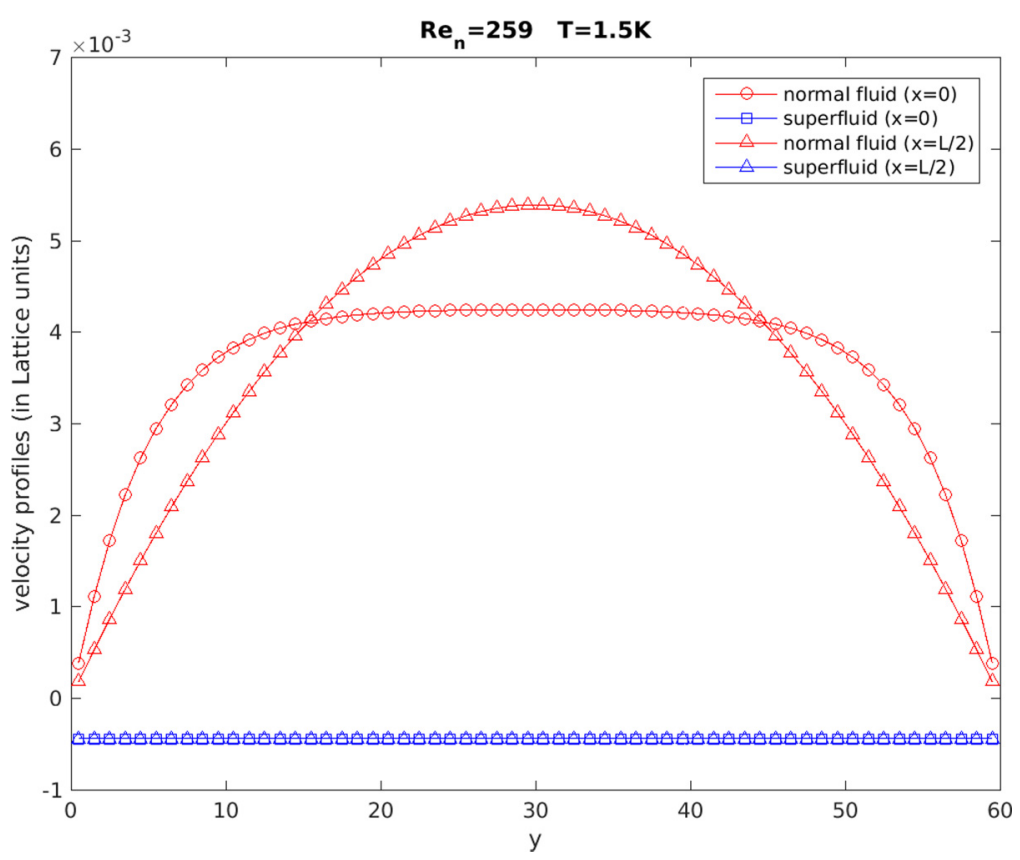

FIG. 12. Velocity profiles at the hot entry $(x=0)$ and in the fully developed regime $(x=L / 2)$. The superfluid profile does not evolve in the normal-fluid entrance region and remains flat.

and

$$
L_{\mathrm{C}}=\frac{p_{\infty}(L)-p(L)}{p_{\infty}^{\prime}}=\frac{1}{p_{\infty}^{\prime}} \int_{x_{\text {mid }}}^{L}\left[p_{\infty}^{\prime}-p^{\prime}(x)\right] d x
$$

where $V_{n}$ is the mean normal-fluid velocity and $x_{\text {mid }}$ is an arbitrary abscissa in the fully developed region. Let us recall that $p_{\infty}^{\prime}$ is the pressure gradient in the fully developed regime (in the center region of the pipe).

The pressure-drop-based entrance lengths $L_{\mathrm{H}}$ estimated for the simulations at $T=1.5 \mathrm{~K}$ are shown in Fig. 11. We found that $L_{\mathrm{H}}$ is typically two times shorter than the entrance length defined from the centerline velocity or the wall shear stress but behaves similarly as a function of the Reynolds number:

$$
2 L_{\mathrm{H}} \simeq L_{v} \propto \operatorname{Re}_{\mathrm{n}} .
$$

\section{B. Normal-fluid versus superfluid entrance lengths}

The entrance lengths $L_{\mathrm{C}}$ and $L_{\mathrm{H}}$ have been estimated in simulations with temperature $1.3 \mathrm{~K} \leqslant$ $T \leqslant 1.96 \mathrm{~K}$ corresponding to density ratio $21.2 \geqslant \rho_{s} / \rho_{n} \geqslant 1.02$ (see Table II). The Reynolds numbers of the simulated flows $\mathrm{Re}_{\mathrm{n}} \lesssim 800$. After a transient stage, a stationary regime (in the statistical sense) establishes in the pipe. The entrance lengths have been estimated from the pressure

TABLE III. Consistency check of the fully developed regime for the simulations at $T=1.5 \mathrm{~K}$ in a pipe of aspect ratio $L / 2 h=30$.

\begin{tabular}{lccccccr}
\hline \hline $\operatorname{Re}_{\mathrm{n}}$ & 32 & 65 & 129 & 259 & 518 & 777 & 1035 \\
$\frac{3 \mu_{n} V_{n}}{h^{2}} / p_{\infty}^{\prime}$ & 1.00 & 1.00 & 1.00 & 1.00 & 1.05 & 1.16 & 1.28 \\
\hline \hline
\end{tabular}




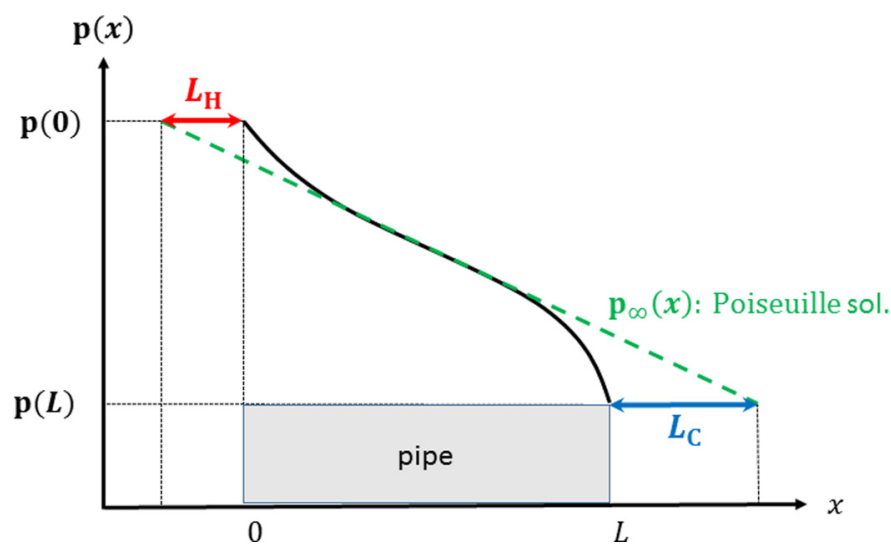

FIG. 13. Sketch of the definition of entrance lengths for the normal fluid, $L_{\mathrm{H}}$, and the superfluid, $L_{\mathrm{C}}$. The continuous black curve represents the pressure drop along the pipe (except the reservoirs) obtained from simulations: $p(x)$ for $0 \leqslant x \leqslant L$. The green dashed line represents the (linear) Poiseuille solution $p_{\infty}(x)$ associated with the pressure gradient measured in the fully developed region. The extrapolation of the Poiseuille solution until $p(0)$ and $p(L)$ allows us to define $L_{\mathrm{H}}$ and $L_{\mathrm{C}}$ as $p_{\infty}\left(-L_{\mathrm{H}}\right)=p(0)$ and $p_{\infty}\left(L+L_{\mathrm{C}}\right)=p(L)$, respectively.

field averaged in time according to Eqs. (32) and (33). One shall see later that the flow may experience local unsteadiness near the cold end at high Reynolds numbers.

The robustness of the results have been checked systematically. In addition to usual test of convergence and resolution, the fulfillment of Eq. (31) with a tolerance smaller than $10 \%$ has been checked to make sure that the simulated pipe was long enough for the establishment of a fully developed region in the central region. This is mandatory to ensure that entrance lengths at the hot and cold ends are independent. We have also verified that the artificial superfluid viscosity with $v_{s} / v_{n}=0.01$ had no significant impact on entry effects.

Figure 14 shows the ratio $L_{\mathrm{C}} / L_{\mathrm{H}}$ as a function of the Reynolds number for various temperatures. The superfluid entrance length rapidly exceeds the normal-fluid entrance length as $\mathrm{Re}_{\mathrm{n}}$ increases. We shall see later that the superfluid entrance length is related to the presence of a superfluid boundary layer. This boundary layer becomes unsteady as the Reynolds number increases, what is indicated by the filled symbols in Fig. 14 . The ratio $L_{\mathrm{C}} / L_{\mathrm{H}}$ rapidly increases at small Reynolds number but seems to saturate when the superfluid boundary layers becomes unstable. The value of the plateau increases as the temperature decreases, or equivalently as the relative superfluid density increases. In this asymptotic regime, the superfluid entrance length exceeds by typically one decade the classical entrance length (observed for the normal fluid at the opposite end). This is a key result of this paper. We shall focus in the next section on the nature of the flow near the cooling reservoir to understand the development of superfluid boundary layers.

It is important to recall here that our entrance lengths are related to the excess of pressure drop due to entry effects. Namely, they are defined as the length of a developed Poiseuille flow (with the same flow rate) that would experience exactly this excess of pressure drop. Equivalently, suppose that one measures the pressure drop between both ends of a duct and evaluates the length of the pipe according to the Poiseuille solution, therefore disregarding entry effects. Our entrance length then corresponds to the overestimation of the pipe length that is made by ignoring entry effects. In that sense, one may consider that our "entrance length" corresponds to a virtual effective extension of the pipe, as sketched in Fig. 13. Unlike previous definitions, our entrance lengths do not correspond to lengths measured on profiles inside the pipe. In principle, very intense pressure drop near the cooling reservoir can lead to an entrance length exceeding the length of the pipe. However, since 


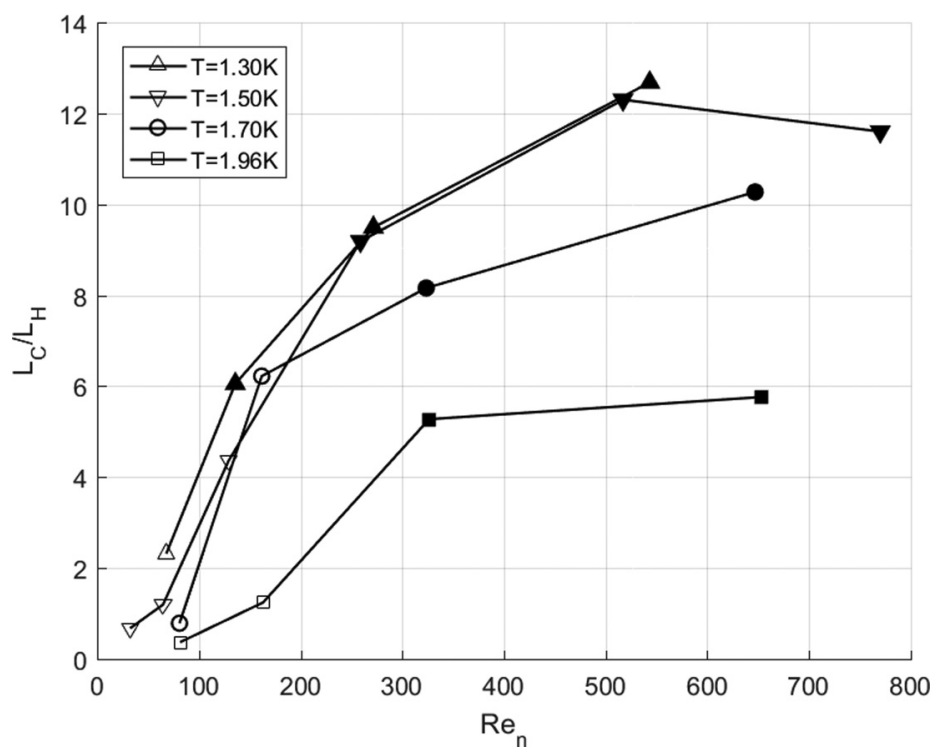

FIG. 14. The ratio of entrance lengths for the superfluid $\left(L_{\mathrm{C}}\right)$ and normal fluid $\left(L_{\mathrm{H}}\right)$ as the function of the Reynolds number $\operatorname{Re}_{\mathrm{n}}$ of the flow. The open symbols indicate that the superfluid boundary layer in the cold entrance region is laminar, whereas filled symbols indicate unsteadiness of the superfluid boundary layer.

this effect is very localized in real space near the cool entrance, it does not exclude the achievement of a fully developed regime in the central region of the pipe.

\section{VELOCITY PROFILES NEAR THE COOLING RESERVOIR}

In this section, simulations at different Reynolds numbers and temperatures are used to illustrate the nature of the flow near the cooling reservoir. Entrance lengths are quantitatively different when the temperature is changed. However, the nature of the flow as a function of the Reynolds number remains qualitatively the same for all temperatures, indicating that the same underlying mechanisms operate. This is now detailed.

At low Reynolds number, the whole counterflow is steady. As a representative configuration, the flow at $T=1.5 \mathrm{~K}\left(\rho_{s} / \rho_{n} \simeq 8\right)$ and Reynolds number $\operatorname{Re}_{\mathrm{n}}=65$ is considered. The streamlines of both components of He-II near the cooling reservoir are displayed in Fig. 15. As the normal fluid flows into the reservoir, its transformation into superfluid is responsible for the development of two

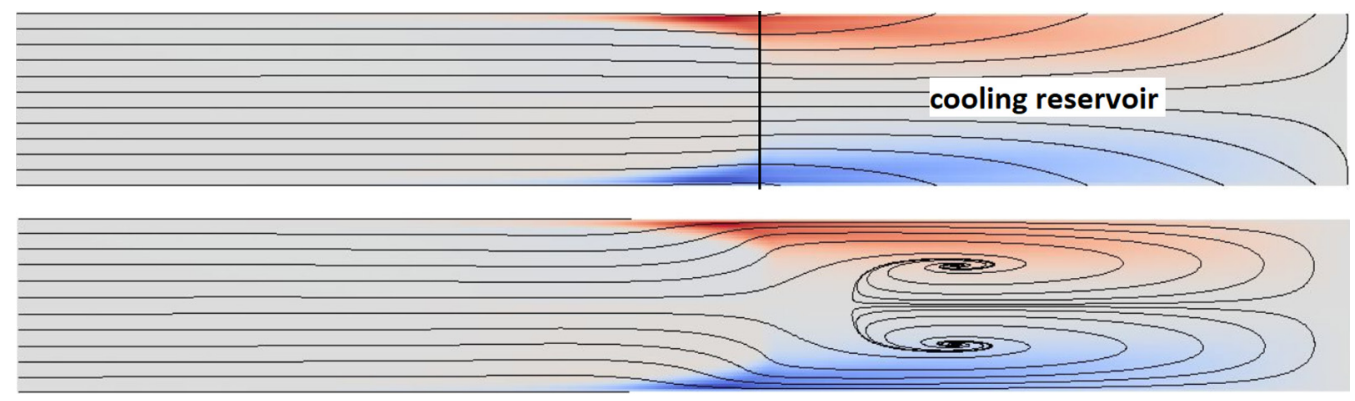

FIG. 15. Streamlines of the normal-fluid (top) and superfluid (bottom) velocities near the cooling reservoir at $T=1.5 \mathrm{~K}$ and $\operatorname{Re}_{\mathrm{n}}=65$. The flow is steady. The superfluid vorticity is represented in the background with an arbitrary color map from blue (negative values) to red (positive values). 


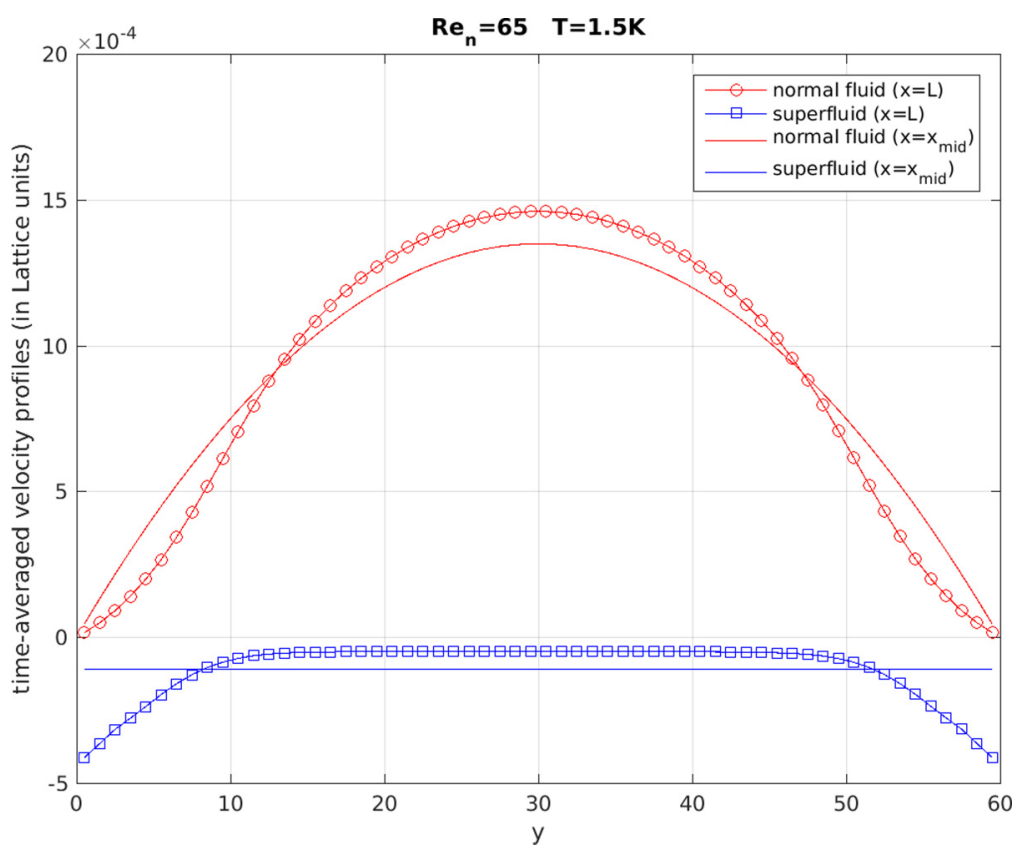

FIG. 16. Velocity profiles of both components of He-II at the entrance of the cooling reservoir $(x=L)$ and in the fully developed region $\left(x=x_{\text {mid }}\right)$ in which superfluid boundary layers have vanished.

large recirculation eddies of superfluid. The resulting current eventually re-enters into the pipe along the side walls. This results in some production of superfluid vorticity along the walls, which is then rapidly damped inside the pipe.

The concentration of vorticity near the walls may be viewed as superfluid boundary layers and interpreted as a signature of superfluid entrance effects. It is observed in Fig. 16 that the fully developed regime is recovered with a Poiseuille profile for the normal fluid and a flat profile for the superfluid as soon as the superfluid boundary layers have vanished. On the contrary, the normal fluid exhibits a tail-flattened profile in the vicinity of the cooling reservoir and the superfluid shows raising tails. In Fig. 17, one observes that superfluid entrance effects are also associated with a significant increase of the pressure drop compared to the Poiseuille pressure drop (in the fully developed regime). This effect extends over about $1.5 \times 2 h$ at $\operatorname{Re}_{\mathrm{n}}=65$ in qualitative agreement with the length of the superfluid boundary layers in Fig. 15.

To evidence the role of the mutual friction in superfluid entrance effects, the same configuration has been run again without mutual coupling $\left(B=B^{\prime}=0\right)$. In that situation, the superfluid boundary layers spreads over the whole pipe and eventually become unstable when reaching the opposite end (see Fig. 18). This comparison clearly evidences the essential role played by mutual friction in the phenomenology of superfluid entry effect, in the same way as viscosity in the entry effect of classical laminar flows.

At higher Reynolds number, superfluid boundary layers undergo instabilities. The counterflow at $T=1.3 \mathrm{~K}\left(\rho_{s} / \rho_{n} \simeq 21\right)$ and $\operatorname{Re}_{\mathrm{n}}=138$ is chosen as a representative situation in Fig. 19. In Fig. 20, the velocity profiles near the cooling reservoir exhibit the same features as before, in particular the flat-tailed profile for the normal fluid velocity, but amplified. The pressure loss near the cooling zone is also greatly enhanced (see Fig. 17). This is fully consistent with the significant increase of the superfluid entrance length previously reported in Fig. 14. 


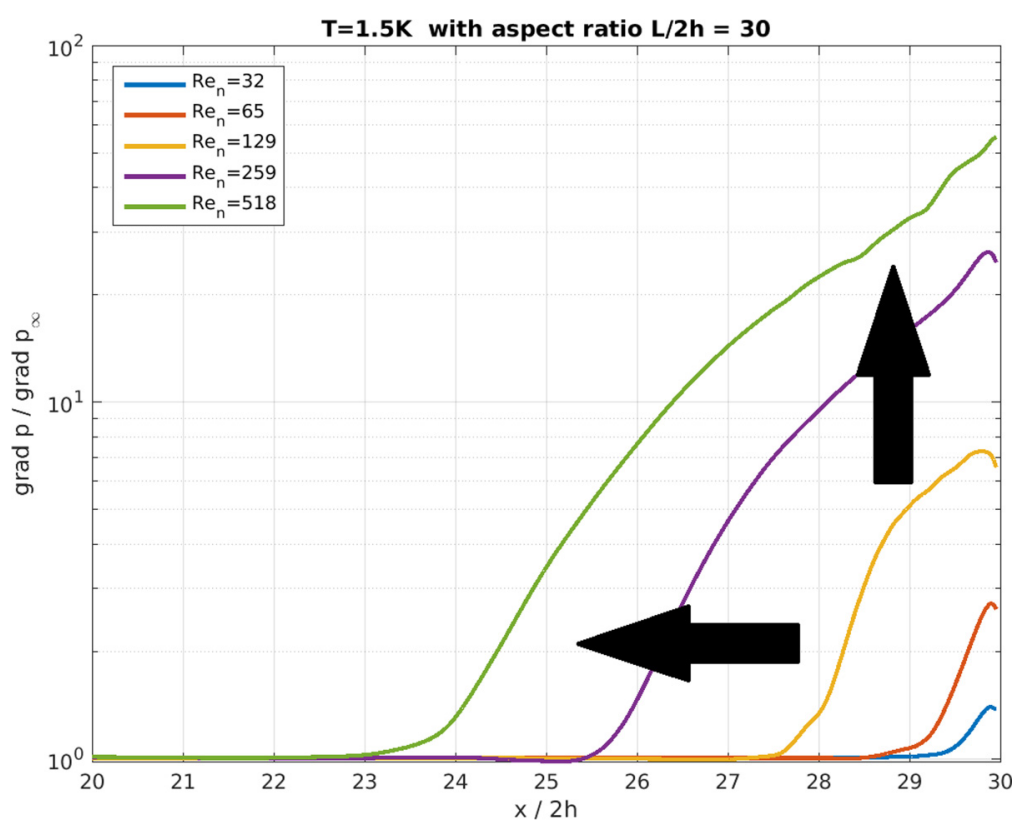

FIG. 17. Evolution of the normalized mean pressure gradient along the centerline of the pipe. A significant pressure drop is experienced by the flow near the cooling zone. The extension and the amplitude of the pressure drop increase with the Reynolds number.

\section{IMPLICATION ON OUR UNDERSTANDING OF THE TRANSITIONS T1 AND T2}

\section{A. A correlation for the superfluid entrance length}

We now come back to the apparent contradiction between experiments presented in the first section of this paper. It is clear from Fig. 3 that all experiments performed using pipes of aspect ratio larger than 550 report consistent thresholds for the $T 1$ transition, whereas experiments with aspect ratio smaller than 140 exhibit widely scattered thresholds. In this section, we shall see that this qualitative finding is supported by our simulations on quantitative grounds.

To confront entrance-length effects with the diversified experimental conditions, an analytical model is required to account for the temperature and Reynolds number dependencies of $L_{\mathrm{C}}$. One possibility would be to model the physics of the superfluid entry effect, and validate this physical model with the present set of simulations. Unfortunately, this approach turns out to be delicate and beyond the scope of the present study, due to the entanglement of the various contributions to $L_{\mathrm{C}}$. We rather adopt an empirical approach now detailed.

The experimental data of Fig. 2 and 3 have been obtained for $\operatorname{Re}_{s}<45$, which corresponds here to $\mathrm{Re}_{n}<400$. Thus, the high-Reynolds-number saturation reported in Fig. 14 is hardly reached in these experimental data sets. Therefore, we will focus on the behavior preceding this apparent saturation, where $L_{\mathrm{C}} / L_{\mathrm{H}}$ is Reynolds number dependent. The normal fluid entrance length $L_{\mathrm{H}}$ was found inversely proportional to the viscosity coefficient $\mu_{n}$, which controls the entry effect at the hot end. At the cold end, mutual friction was found to play a central role in damping the entry effect, so one can expect $L_{\mathrm{C}}$ to be inversely proportional to the mutual friction coefficient $B$ (the other mutual

FIG. 18. When the mutual friction is turned off, superfluid boundary layers extend along the whole pipe. 


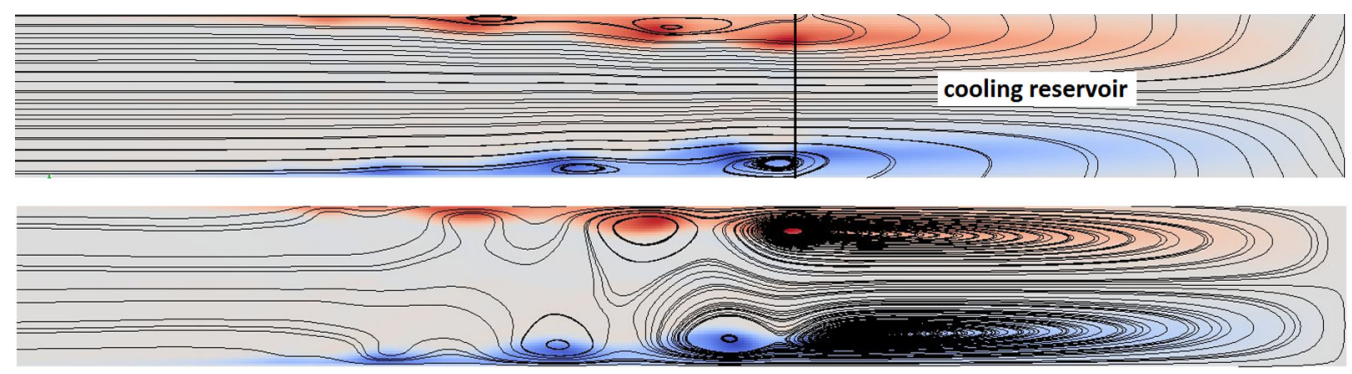

FIG. 19. Streamlines of the normal-fluid (top) and superfluid (bottom) velocities near the cooling reservoir at $T=1.3 \mathrm{~K}$ and $\mathrm{Re}_{\mathrm{n}}=136$. The superfluid vorticity is represented in the background with an arbitrary colormap from blue (negative values) to red (positive values).

friction term is proportional to $B^{\prime}$ and smaller in magnitude). Thus, to compensate the ratio $L_{\mathrm{C}} / L_{\mathrm{H}}$ for the temperature dependence of $\mu_{n}$ and $B$, the alternative ratio

$$
\Gamma=\frac{L_{\mathrm{C}}}{L_{\mathrm{H}}} \frac{B \rho \kappa}{\mu_{n}}
$$

appears to be more relevant. In order to keep the ratio dimensionless, we also took advantage of the limited temperature dependence of $\rho \kappa$ to use this quantity as a prefactor. The dimensionless ratio $\Gamma$ obtained in the simulations are plotted versus ${ }_{s}$ in Fig. 21 by rescaling both abscissa and ordinate by some arbitrary power of $\rho_{s} / \rho$ chosen to obtain the best collapse over a wide region. The collapse

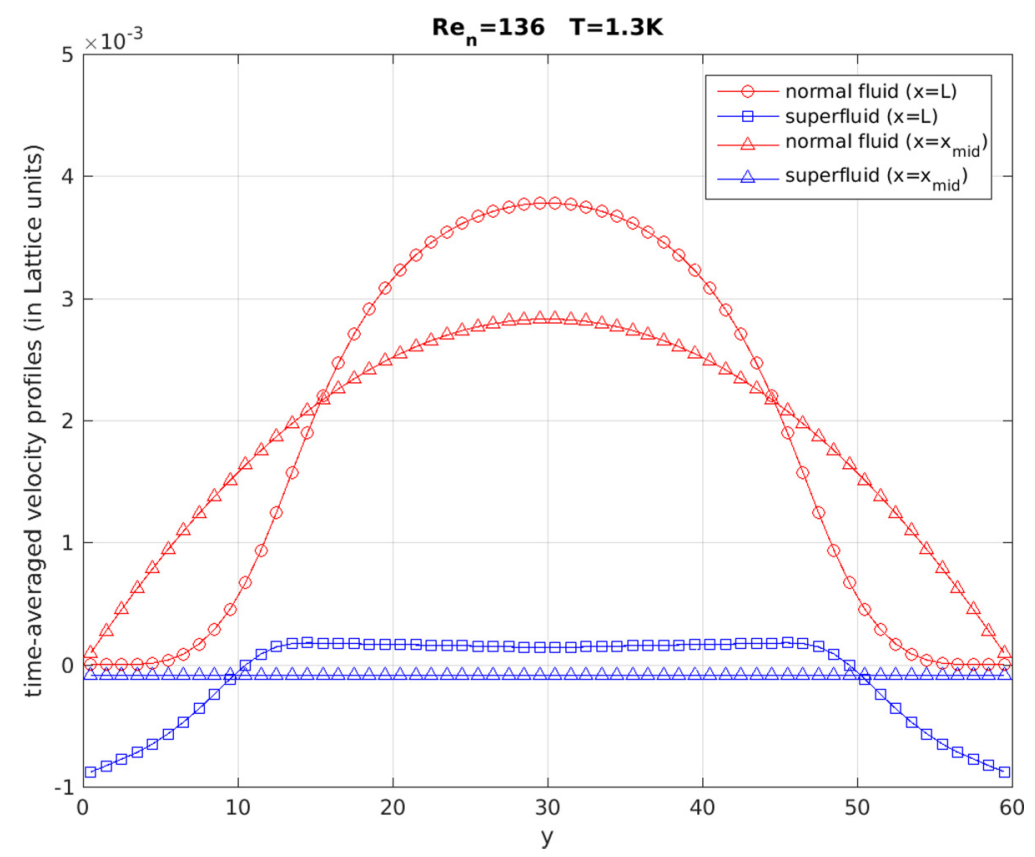

FIG. 20. Time-averaged velocity profiles of both components of He-II at the entrance of the cooling zone $(x=L)$ and in the fully developed region $\left(x=x_{\text {mid }}\right)$ for the same flow as in Fig. 19. 


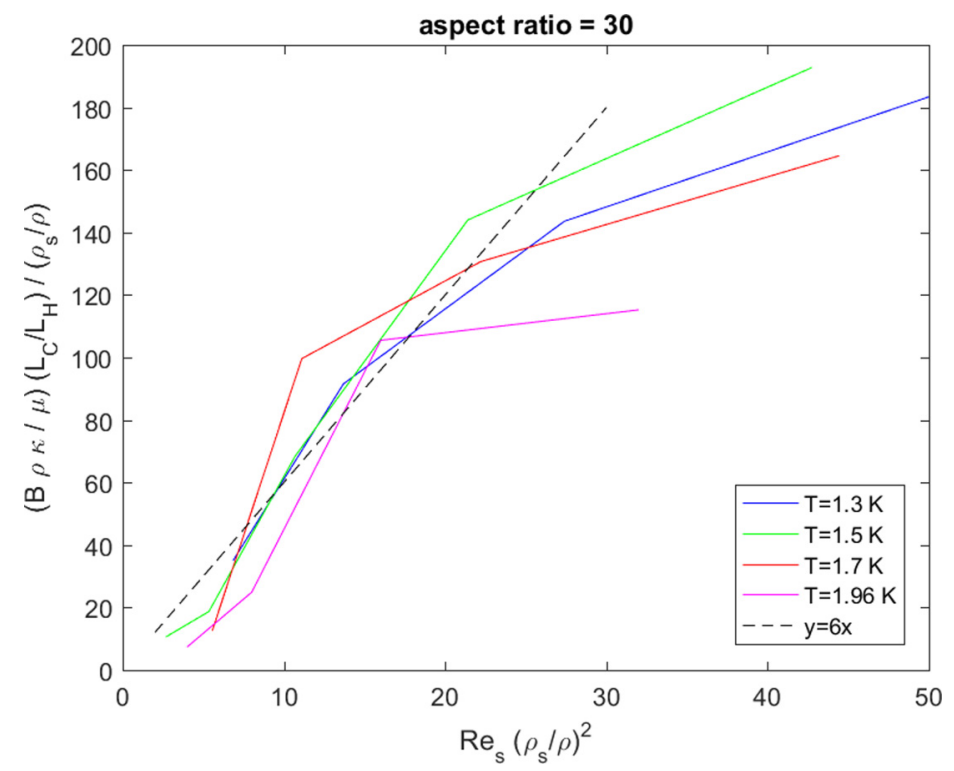

FIG. 21. Rescaled version of Fig. 14. The dashed line is a fit used to discuss the $T 1$ transition.

suggests the following empirical fit

$$
\frac{L_{\mathrm{C}}}{L_{\mathrm{H}}} \frac{B \rho \kappa}{\mu} \frac{\rho}{\rho_{s}} \simeq G\left(\operatorname{Re}_{s}\left(\frac{\rho_{s}}{\rho}\right)^{2}\right),
$$

where $G($.$) is a monotonic function. Using the crude linear approximation G(x)=6 x$ for $0<x<30$ (see the dashed line in Fig. 21), the empirical law for $L_{\mathrm{C}} / L_{\mathrm{H}}$ becomes

$$
\frac{L_{\mathrm{C}}}{L_{\mathrm{H}}} \simeq \frac{6 \mu}{\rho \kappa B}\left(\frac{\rho_{s}}{\rho}\right)^{3} \operatorname{Re}_{s} .
$$

Equations (36) and (37) should just be considered as convenient fits within $1.3 \mathrm{~K} \leqslant T \leqslant 1.96 \mathrm{~K}$ without physical significance. For example, there is no reason for the $\rho_{s} / \rho$ dependence to be the same at low $\mathrm{Re}_{s}$, where the superfluid boundary layer is stable, and at large $\mathrm{Re}_{s}$, where it is unstable. Together with Eqs. (34) and (30), we are now able to estimate $L_{\mathrm{C}}$ over a broad range of experimental conditions as

$$
L_{\mathrm{C}} \simeq \frac{6 \mu}{B \rho \kappa}\left(\frac{\rho_{s}}{\rho}\right)^{3} \operatorname{Re}_{s} \times \frac{1}{2} 0.05 \operatorname{Re}_{\mathrm{n}} d,
$$

which eventually yields

$$
L_{\mathrm{C}} \simeq \frac{d}{7 B}\left(\frac{\rho_{s}}{\rho}\right)^{4} \mathrm{Re}_{s}^{2}
$$

\section{B. On the $T 3$ transition}

In channels with a high-aspect-ratio rectangular cross section, it has been argued that the $T 1$ and $T 2$ transitions are replaced by a unique transition, called $T 3$ [2]. Experimental evidences for a unique transition in such geometry are few, and most of them were provided by the group of Tough, who reports that the "critical region is found to be poorly defined" [32]. Today, this $T 3$ transition is not better understood than $T 1$ and $T 2$. Still, it is interesting to discuss if our two-dimensional (2D) 
simulations would be more representative of these high-aspect-ratio rectangular channels than of circular or squared channels, as assumed so far.

In a $2 \mathrm{D}$ channel, the flow is sensitive to only one transverse length scale (the channel height $2 h$, with our notation) and instabilities may be indexed by a Reynolds number proportional to this length scale. Physically, such a 2D geometry corresponds to the limit of a 3D channel between two infinitely close free-slip walls. In a 3D channel of rectangular cross section $2 h \times w$ with $w \gg 2 h$, the first flow instability is expected to develop along the longest dimension, that is when the Reynolds number based on the length scale $w$ reaches some threshold. However, this threshold inevitably depends on the other length scale $(2 h)$ because the flow feels the confinement along this other direction. Thus, both length scales $2 h$ and $w$ remain relevant in the determination of the first instability, which differs from the 2D situation mentioned previously. On the contrary, flows in channels with circular (resp., squared) cross section are characterized by a single characteristic transverse length scale: the channel diameter (resp., square side). In this respect, the 2D model is more relevant to these type of flows than to 3D channel with high-aspect-ratio cross section.

\section{On the $T 1$ transition}

To determine experimentally the threshold of the $T 1$ transition without entry effect contamination, the pipe length $L$ should be much longer than the sum of the entrance lengths, i.e.,

$$
L \gg L_{\mathrm{H}}+L_{\mathrm{C}} \simeq L_{\mathrm{C}}
$$

Using Eq. (39), we can evaluate the required aspect ratio $L / d$ such that $L_{\mathrm{C}} / L=10 \%$ and $L_{\mathrm{C}} / L=1 \%$ at $1.2 \mathrm{~K}\left(B=1.55\right.$ and $\left.\rho_{s} / \rho=97 \%\right), 1.35 \mathrm{~K}\left(B=1.35\right.$ and $\left.\rho_{s} / \rho=94 \%\right)$, and $1.7 \mathrm{~K}\left(B=1.1\right.$ and $\left.\rho_{s} / \rho=76 \%\right)$. The results are plotted in Fig. 3 with continuous and dashed lines respectively for the $10 \%$ and $1 \%$ criteria. These lines allow a quantitative interpretation of the separation between the two groups of experiments. All the consistent determination of the $T 1$ threshold are obtained for experiments with an estimated entrance length smaller than typically $\sim 1 \%$ of the pipe length. For all the other experiments, with inconsistent $T 1$ thresholds, the estimated entrance lengths extend over more than $\sim 10 \%$ of the pipe or channel. Surely, these $1 \%$ and $10 \%$ criteria should be considered as indicative. They would have been different if we had used a different definition of the entrance lengths. Nevertheless, the results of the present numerical study support quantitatively our explanation for the contradictory thresholds reported for the $T 1$ transitions since the 1960s.

\section{On the $T 2$ transition}

The $T 2$ transition is sometimes detected experimentally from the increase of pressure drop resulting from the transition to turbulence of the normal fluid. It is interesting to observe how the entry effect could mimic a $T 2$ transition. Indeed, below the $T 1$ transition, the pressure drop $\Delta \mathrm{p}$ along the pipe is by definition of $L_{\mathrm{C}}$ and $L_{\mathrm{H}}$ :

$$
\Delta p=p_{\infty}^{\prime}\left(L+L_{\mathrm{C}}+L_{\mathrm{H}}\right),
$$

where the pressure gradient $p_{\infty}^{\prime}$ was defined by Eq. (31). For $\operatorname{Re}_{n}>200$, a rough estimate of the entry lengths ratio is $L_{\mathrm{C}} / L_{\mathrm{H}} \simeq 10$ (see Fig. 14). As long as the normal fluid remains laminar, the entrance length $L_{\mathrm{H}}$ can be estimated by using Eqs. (34) and (30). For a given temperature, simple algebra based on these estimates leads to the proportionality

$$
\Delta p \sim \operatorname{Re}_{\mathrm{n}}\left[1+\frac{d}{L} \frac{\operatorname{Re}_{\mathrm{n}}}{4}\right] .
$$

The quadratic term $\mathrm{Re}_{\mathrm{n}}{ }^{2}$, which is associated to the entry effects, becomes larger than the linear term for $\operatorname{Re}_{n}>4 L / d$. This could erroneously be interpreted as the pressure-drop increase associated with the $T 2$ transition. For example, in a pipe of aspect ratio of $L / d=400$, this pressure increase 


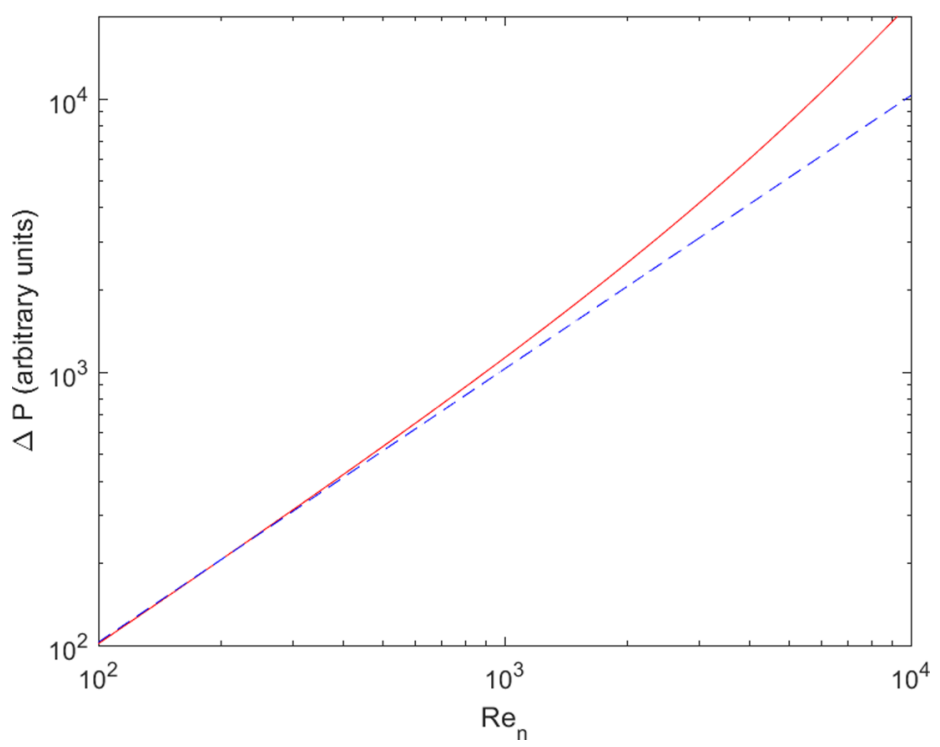

FIG. 22. Red curve: False signature of the $T 2$ transition caused by entrance effects in a pipe of aspect ratio 400. The normal fluid flow is assumed laminar in its entry and developed regions. The dashed blue line is an eye guide.

becomes noticeable around $\operatorname{Re}_{n}=1000$, as illustrated in Fig. 22, that is, at a Reynolds number typical of the laminar to turbulent transition in a pipe.

Therefore, entry effects can mimic the signature of the $T 2$ transition, or conversely, some pressure increases may have been misinterpreted as $T 2$ transitions even with pipes of large (but not sufficiently large) aspect ratio. Our study shows that using pipe and channel of very large aspect ratio can also be important when studying the $T 2$ transition. In this perspective, another analysis of literature experimental data published on the $T 2$ transition could help to understand the controversy on this second transition as well.

\section{CONCLUSION AND PERSPECTIVES}

We have introduced a quantitative definition of the entrance length based on an measurable quantity: the pressure drop along the pipe (or channel). This definition can be applied in a similar fashion to both entries of a counterflow, and it does not rely on some arbitrary convergence threshold, unlike classical definitions. Based on this definition and from simulations below the $T 1$ transition, it is found that the entrance length on the superfluid entry side can be more than one decade longer than on the normal entry side. Surely, if we had used other definitions for the entrance lengths $L_{\mathrm{C}}$ and $L_{\mathrm{H}}$ and ratio $L_{\mathrm{C}} / L_{\mathrm{H}}$ would have been different. Different models of reservoir (in particular with geometrical discontinuities and with surface rather than volume heating and cooling) could also lead to different values, with possibly much larger $L_{C}$. Still, the physical processes that have been identified are expected to be independent of these choices, because the strong superfluid entrance effects originate from two simple features of counterflow, (1) the absence of superfluid viscosity and (2) the long persistence of superfluid vorticity continuously fueled into the pipe by the cold reservoir, which distort both the normal and superfluid flows in the cooling-side entrance zone.

We address the issue of the experimental conditions to determine the threshold of the $T 1$ transition and demonstrate the importance of the pipe aspect ratio. A comparative study of results published over the past few decades, combined with a simple model of the entrance effect backed up by simulations, shows that the pipe length should exceed several hundreds times its diameter to resolve the $T 1$ transition while remaining free of artifacts from end effects. When this aspect ratio criterion 
is verified, the published data for the $T 1$ transition threshold become consistent again, which is not the case for the data obtained at the lowest aspect ratio. This interpretation provides a solution to a 50 -year-old open problem on one of the most simple hydrodynamics transitions of superfluids. We hope the present work will help to sort among existing experimental data sets when testing models of the $T 1$ transition. The origin of the $T 1$ instability itself was not addressed in this work and remains an open question. This question may not be addressable with the HVBK model, which supposes the pre-existence of background vortices.

We showed that the entrance length effect can also mimic a $T 2$ transition, even in pipes of aspect ratio of a few hundreds. This observation may contribute to the difficult and controversial topic of the $T 2$ transition by allowing to discard some suspicious experimental data sets as well.

Thanks to the recent progresses in flow visualization techniques, new counterflow experiments in $\mathrm{cm}$-sized channel have been developed in recent years ([81,82]) to explore the velocity profiles and ultimately to understand the nature of flow transitions. The present work shows that special attention is deserved when designing the reservoir and its connection with the counterflow channel (not to mention the channel aspect ratio issue), at least for studies below the $T 1$ transition. For instance, the amount of vorticity entering in the channel should be reduced by proper flow conditioning and spatially distributed reservoir cooling. An excessive entry of quantum vortices will indeed generate far-reaching distortions of the superfluid and normal velocity fields (e.g., with production of flat-tailed normal fluid velocity profiles and raised-tail profile for the superfluid) and differ in the settling of the steady state in the channel.

In classical hydrodynamics, the understanding of the laminar to turbulent transition in pipes has been a subject of research for more than a century [83] and the importance of proper flow conditioning appeared quite early. In superfluid helium, the situation is expected to be even more complicated due to the presence of two fluids and ill-understood boundary layer interactions. As stated by Schwarz in 1992, "the critical velocities represent a much more complicated problem than the fully developed vortex tangle, and that they require consideration of a variety of detailed factors. We are certainly a long way from a full understanding (p. 3344)" [39]. In this study, we found that classical hydrodynamics design rules are not sufficient as far as pipe aspect ratio and penetration depth of small-scale vorticity is concerned. The memory effect associated with remanent superfluid vortices is another example of a limitation of classical design rule. To fully understand the $T 1$ and $T 2$ transitions, the prior development of an engineering of flow conditioning of quantum fluids will be probably needed.

We hope that this work has also paved the way for use of the lattice Boltzmann method for finite-temperature simulation of superfluid flows. In particular, this numerical method allows us to take into account complex geometry of boundary layer, which opens the way for the study of wakes, friction, and lift forces in He-II.

\section{ACKNOWLEDGMENTS}

The simulations have been performed by using the local HPC facilities at Ens de Lyon (PSMN) and were mainly supported by the Auvergne-Rhône-Alpes region (Grant No. CPRT07-13 CIRA), the project Equip@Meso (ANR-10-EQPX-29-01) and ANR Ecouturb (ANR-16-CE30-0016). J.B. has been supported by a Ph.D. grant from the Auvergne-Rhône-Alpes region (ARC Energies 2012 program). We would like to thank Alessandro de Rosis for insightful comments and his help in designing the LB scheme.

[1] W. F. Vinen, Mutual friction in a heat current in liquid helium II. II experiments on transient effects, Proc. R. Soc. London, Ser. A 240, 128 (1957). 


\section{J. BERTOLACCINI, E. LÉVÊQUE, AND P.-E. ROCHE}

[2] J. T. Tough, Superfluid Turbulence (North-Holland, Amsterdam, 1982), Vol. 8, Chap. 3, pp. 133-219.

[3] S. K. Nemirovskii and W. Fiszdon, Chaotic quantized vortices and hydrodynamic processes in superfluid helium, Rev. Mod. Phys. 67, 37 (1995).

[4] R. J. Donnelly, Quantized Vortices in Helium-II, Cambridge Studies in Low Temperature Physics (Cambridge University Press, Cambridge, UK, 1991).

[5] S. W. Van Sciver, Helium Cryogenics, International Cryogenics Monograph Series (Springer, Berlin, 2012).

[6] Superfluid Helium, edited by J. F. Allen (Academic Press, New York, 1966).

[7] L. D. Landau, The theory of superfluidity of helium II, J. Phys. (USSR) 5, 71 (1941).

[8] S. V. Iordanskii, Vortex ring formation in a superfluid, Sov. Phys. JETP 21, 467 (1965).

[9] J. S. Langer and M. E. Fisher, Intrinsic Critical Velocity of a Superfluid, Phys. Rev. Lett. 19, 560 (1967).

[10] V. I. Kruglov, Critical velocities and two mechanisms of transition in superfluid liquid 44He, Phys. Lett. A 375, 4058 (2011).

[11] P. P. Craig, Critical velocities in superfluid He II, Phys. Lett. 21, 385 (1966).

[12] B. K. Jones, High speed vortices in helium, Phys. Rev. 177, 292 (1969).

[13] R. P. Feynman, Progress in Low Temperature Physics (North-Holland, Amsterdam, 1955), Vol. 1.

[14] V. P. Peshkov, Critical velocities in superfluid helium, J. Exptl. Theoret. Phys. (USSR) 40, 379 (1961).

[15] J. C. Fineman and C. E. Chase, Energy of a vortex ring in a tube and critical velocities in liquid helium II, Phys. Rev. 129, 1 (1963).

[16] W. Glaberson and R. Donnelly, Growth of pinned quantized vortex lines in helium II, Phys. Rev. 141, 208 (1966).

[17] C. E. Swanson and R. J. Donnelly, Vortex dynamics and scaling in turbulent counterflowing helium II, J. Low Temp. Phys. 61, 363 (1985).

[18] K. W. Schwarz, Three-dimensional vortex dynamics in superfluid 4He: Homogeneous superfluid turbulence, Phys. Rev. B 38, 2398 (1988).

[19] C. F. Barenghi, D. C. Samuels, and G. H. Bauer, Superfluid vortex lines in a model of turbulent flow, Phys. Fluids 9, 2631 (1997).

[20] A. Fetter, Vortex Rings and the Critical Velocity in Helium II, Phys. Rev. Lett. 10, 507 (1963).

[21] R. K. Childers and J. T. Tough, Helium II thermal counterflow: Temperature- and pressure-difference data and analysis in terms of the vinen theory, Phys. Rev. B 13, 1040 (1976).

[22] M. Mongiovi and D. Jou, Generalization of Vinen's equation including transition to superfluid turbulence, J. Phys.: Condens. Matter 17, 4423 (2005).

[23] W. M. Van Alphen, G. J. Van Haasteren, R. de Bruyn Ouboter, and K. W. Taconis, The dependence of the critical velocity of the superfluid on channel diameter and film thickness, Phys. Lett. 20, 474 (1966).

[24] J. Tough, Turbulence in a Rotating Superfluid, Phys. Rev. Lett. 44, 540 (1980).

[25] F. A. Staas, K. W. Taconis, and W. M. Van Alphen, Experiments on laminar and turbulent flow of He II in wide capillaries, Phys. (Amsterdam, Neth.) 27, 893 (1961).

[26] D. F. Brewer and D. O. Edwards, Heat conduction by liquid helium II in capilliary tubes, I: Transition to supercritical conduction, Philos. Mag. 6, 775 (1961).

[27] D. F. Brewer and D. O. Edwards, Heat conduction by liquid helium II in capillary tubes, III: Mutual friction, Philos. Mag. 7, 721 (1962).

[28] C. Chase, Thermal conduction in liquid helium II, I: Temperature dependence, Phys. Rev. 127, 361 (1962).

[29] C. Chase, Thermal conduction in liquid helium II, II: Effects of channel geometry, Phys. Rev. 131, 1898 (1963).

[30] R. K. Childers and J. T. Tough, Critical Velocities as a Test of the Vinen Theory, Phys. Rev. Lett. 31, 911 (1973).

[31] D. R. Ladner and J. T. Tough, Helium II thermal counterflow at large heat currents: Profound effects of geometry, Phys. Rev. B 17, 1455 (1978).

[32] D. R. Ladner and J. T. Tough, Temperature and velocity dependence of superfluid turbulence, Phys. Rev. B 20, 2690 (1979).

[33] K. P. Martin and J. T. Tough, Evolution of superfluid turbulence in thermal counterflow, Phys. Rev. B 27, 2788 (1983). 
[34] A. G. F. Dorscheidt, H. van Kempen, P. Wyder, and T. H. K. Frederking, Thermal counterflow experiments on superfluid helium at temperatures close to $T_{\lambda}$, Phys. Rev. B 31, 5722 (1985).

[35] L. Skrbek, A flow phase diagram for helium superfluids, JETP Lett. 80, 474 (2004).

[36] D. J. Melotte and C. F. Barenghi, Transition to Normal Fluid Turbulence in Helium II, Phys. Rev. Lett. 80, 4181 (1998).

[37] G. Marees, P. J. M. van der Slot, and H. van Beelen, Subcritical and supercritical nonturbulent flow of mass and entropy in superfluid 4He, Phys. B+C (Amsterdam, Neth.) 144, 209 (1987).

[38] S. S. Courts and J. T. Tough, Transition to superfluid turbulence in 2-fluid flow of he-II, Phys. Rev. B 38, 74 (1988).

[39] K. Schwarz, Effect of Surface Roughness on the Critical Velocities of Superfluid 4He, Phys. Rev. Lett. 69, 3342 (1992).

[40] S. Babuin, M. Stammeier, E. Varga, M. Rotter, and L. Skrbek, Quantum turbulence of bellows-driven ${ }^{4} \mathrm{He}$ superflow: Steady state, Phys. Rev. B 86, 134515 (2012).

[41] E. Varoquaux, Anderson's considerations on the flow of superfluid helium: Some offshoots, Rev. Mod. Phys. 87, 803 (2015).

[42] T. K. Lesniewski, T. H. K. Frederking, and S. W. K. Yuan, On He-II inertia effects in short narrow ducts: Entrance effects associated with boundary lay development, Cryogenics 36, 203 (1996).

[43] M. Yamaguchi, Y. Fujii, M. Kishida, and M. Nakamura, He II thermal counterflow near superfluid turbulent transition, Jpn. J. Appl. Phys. 26, 87 (1987).

[44] E. S. Raja Gopal and S. M. A. Tirmizi, Growth of turbulence in the flow of liquid helium II, Cryogenics 4, 378 (1964).

[45] S. M. Bhagat, P. R. Critchlow, and K. Mendelssohn, Onset and growth of vorticity in liquid helium-II, Cryogenics 4, 166 (1964).

[46] N. H. Ramadan and R. J. Witt, Natural convection in large He II baths, Cryogenics 34, 563 (1994).

[47] H. Tatsumoto, K. Hata, K. Hama, Y. Shirai, and M. Shiotsu, Numerical analysis of two-dimensional steady-state and transient heat transfer in a parallel duct filled with pressurized He II, Cryogenics 44, 273 (2004).

[48] K. W. Schwarz, Three-dimensional vortex dynamics in superfluid ${ }^{4} \mathrm{He}$ : Line-line and line-boundary interactions, Phys. Rev. B 31, 5782 (1985).

[49] M. Tsubota, Capacity of a pinning site for trapping quantized vortices in superfluid 4He, Phys. Rev. B 50, 579 (1994).

[50] C. F. Barenghi, V. S. L'vov, and P.-E. Roche, Experimental, numerical, and analytical velocity spectra in turbulent quantum fluid, Proc. Natl. Acad. Sci. USA 111, 4683 (2014).

[51] C. Soulaine, M. Quintard, H. Allain, B. Baudouy, and R. Van Weelderen, A piso-like algorithm to simulate superfluid helium flow with the two-fluid model, Comput. Phys. Commun. 187, 20 (2015).

[52] R. J. Donnelly and C. E. Swanson, Quantum turbulence, J. Fluid Mech. 173, 387 (1986).

[53] A. Libchaber, Le problème de la vitesse critique dans l'hélium superfluide, J. Phys. Colloques 34, C10 (1973).

[54] H. Yano, Y. Nago, R. Goto, K. Obara, O. Ishikawa, and T. Hata, Critical behavior of steady quantum turbulence generated by oscillating structures in superfluid He-4, Phys. Rev. B 81, 220507 (2010).

[55] D. Kivotides, Spreading of superfluid vorticity clouds in normal-fluid turbulence, J. Fluid Mech. 668, 58 (2011).

[56] R. Aarts and F. de Waele, Numerical investigation of the flow properties of He II, Phys. Rev. B 50, 10069 (1994).

[57] C. F. Barenghi and L. Skrbek, On decaying counterflow turbulence in He II, J. Low Temp. Phys. 146, 5 (2007).

[58] H. Adachi, S. Fujiyama, and M. Tsubota, Steady-state counterflow quantum turbulence: Simulation of vortex filaments using the full Biot-Savart law, Phys. Rev. B 81, 104511 (2010).

[59] A. W. Baggaley and J. Laurie, Thermal counterflow in a periodic channel with solid boundaries, J. Low Temp. Phys. 178, 35 (2015).

[60] S. Yui and M. Tsubota, Counterflow quantum turbulence of He-II in a square channel: Numerical analysis with nonuniform flows of the normal fluid, Phys. Rev. B 91, 184504 (2015). 


\section{J. BERTOLACCINI, E. LÉVÊQUE, AND P.-E. ROCHE}

[61] L. Galantucci, M. Sciacca, and C. F. Barenghi, Coupled normal fluid and superfluid profiles of turbulent helium II in channels, Phys. Rev. B 92, 174530 (2015).

[62] D. Khomenko, P. Mishra, and A. Pomyalov, Coupled dynamics for superfluid 4He in a channel, J. Low Temp. Phys. 187, 405 (2017).

[63] T. Kitamura, K. Shiramizu, N. Fujimoto, Y. F. Rao, and K. Fukuda, A numerical model on transient, two-dimensional flow and heat transfer in He II, Cryogenics 37, 1 (1997).

[64] T. Suekane, M. Sekiguchi, S. Hirai, and T. Okamura, Heat transfer and flow of He II in narrow channels, Cryogenics 43, 125 (2003).

[65] L. Tisza, Transport phenomena in helium II, Nature (London) 141, 913 (1938).

[66] R. N. Hills and P. H. Roberts, Superfluid mechanics for a high density of vortex lines, Arch. Ration. Mech. Anal. 66, 43 (1977).

[67] J. Boussinesq, Théorie Analytique de la Chaleur (Gauthier-Villars, Paris, 1903).

[68] C. E. Swanson, C. F. Barenghi, and R. J. Donnelly, Rotation of a Tangle of Quantized Vortex Lines in He II, Phys. Rev. Lett. 50, 190 (1983).

[69] M. Tsubota, C. F. Barenghi, T. Araki, and A. Mitani, Instability of vortex array and transitions to turbulence in rotating helium II, Phys. Rev. B 69, 134515 (2004).

[70] N. Andersson, T. Sidery, and G. L. Comer, Superfluid neutron star turbulence, Mon. Not. R. Astron. Soc. 381, 747 (2007).

[71] J. A. Geurst, Mutual friction in the laminar flow of superfluid helium II through capillary tubes, Phys. Lett. A 71, 78 (1979).

[72] S. Succi, The Lattice Boltzmann Equation for Fluid Dynamics and Beyond (Clarendon Press, Oxford, UK, 2001).

[73] H. Chen, S. Chen, and W. H. Matthaeus, Recovery of the navier-stokes equations using a lattice-gas Boltzmann method, Phys. Rev. A 45, R5339(R) (1992).

[74] P. L. Bhatnagar, E. P. Gross, and M. Krook, A model for collision process in gases, I: Small amplitude processes in charged and neutral one-component system, Phys. Rev. 94, 511 (1954).

[75] S. Chapman and T. G. Cowling, The Mathematical Theory of Non Uniform Gases (Cambridge University Press, UK, 1991).

[76] Z. Guo, C. Zheng, and B. Shi, Discrete lattice effects on the forcing term in the lattice Boltzmann method, Phys. Rev. E 65, 046308 (2002).

[77] P. J. Dellar, Incompressible limits of lattice Boltzmann equations using multiple relaxation times, J. Comput. Phys. 190, 351 (2003).

[78] M. Geier, A. Greiner, and J. G. Korvink, Cascaded digital lattice Boltzmann automata for high Reynolds number flow, Phys. Rev. E 73, 066705 (2006).

[79] K. N. Premnath and S. Banerjee, Incorporating forcing terms in cascaded lattice Boltzmann approach by method of central moments, Phys. Rev. E 80, 036702 (2009).

[80] R. J. Donnelly and C. F. Barenghi, Observed properties of liquid helium at the saturated vapor pressure, J. Phys. Chem. Ref. Data 27, 1217 (1998).

[81] W. Guo, M. L. Mantia, D. P. Lathrop, and S. W. Van Sciver, Visualization of two-fluid flows of superfluid helium-4, Proc. Natl. Acad. Sci. USA 111, 4653 (2014).

[82] A. Marakov, J. Gao, W. Guo, S. W. Van Sciver, G. G. Ihas, D. N. McKinsey, and W. F. Vinen, Visualization of the normal-fluid turbulence in counterflowing superfluid ${ }^{4} \mathrm{He}$, Phys. Rev. B 91, 094503 (2015).

[83] B. Eckhardt, T. M. Schneider, B. Hof, and J. Westerweel, Turbulence transition in pipe flow, Annu. Rev. Fluid Mech. 39, 447 (2007). 